\title{
Unique folding of precursor microRNAs: Quantitative evidence and implications for de novo identification
}

\author{
STANLEY NG KWANG LOONG ${ }^{1,2}$ and SANTOSH K. MISHRA ${ }^{1,2}$ \\ ${ }^{1}$ Bioinformatics Institute, Matrix, Singapore 138671 \\ ${ }^{2}$ NUS Graduate School for Integrative Sciences and Engineering, Centre for Life Sciences, Singapore 117456
}

\begin{abstract}
MicroRNAs (miRNAs) participate in diverse cellular and physiological processes through the post-transcriptional gene regulatory pathway. Hairpin is a crucial structural feature for the computational identification of precursor miRNAs (pre-miRs), as its formation is critically associated with the early stages of the mature miRNA biogenesis. Our incomplete knowledge about the number of miRNAs present in the genomes of vertebrates, worms, plants, and even viruses necessitates thorough understanding of their sequence motifs, hairpin structural characteristics, and topological descriptors. In this in-depth study, we investigate a comprehensive and heterogeneous collection of 2241 published (nonredundant) pre-miRs across 41 species (miRBase 8.2), 8494 pseudohairpins extracted from the human RefSeq genes, 12,387 (nonredundant) ncRNAs spanning 457 types (Rfam 7.0), 31 full-length mRNAs randomly selected from GenBank, and four sets of synthetically generated genomic background corresponding to each of the native RNA sequence. Our large-scale characterization analysis reveals that pre-miRs are significantly different from other types of ncRNAs, pseudohairpins, mRNAs, and genomic background according to the nonparametric Kruskal-Wallis ANOVA $(p<0.001)$. We examine the intrinsic and global features at the sequence, structural, and topological levels including \% $G+C$ content, normalized base-pairing propensity $P(S)$, normalized minimum free energy of folding MFE(s), normalized Shannon entropy $Q(s)$, normalized base-pair distance $D(s)$, and degree of compactness $F(S)$, as well as their corresponding $Z$ scores of $P(S), M F E(s), Q(s), D(s)$, and $F(S)$. The findings will promote more accurate guidelines and distinctive criteria for the prediction of novel pre-miRs with improved performance.
\end{abstract}

Keywords: precursor microRNAs; minimum free energy of folding; Shannon entropy; Z-scores; second eigenvalue

\section{INTRODUCTION}

MicroRNAs (miRNAs) constitute an abundant class of endogenous and small $(\sim 21-23$-nucleotides $[\mathrm{nt}])$ regulatory ncRNA molecules that mediate post-transcriptionally the production of intracellular proteins in most eukaryotes (Ambros 2001; Bartel 2004; Mallory and Vaucheret 2004). The pioneers lin-4 and let-7 miRNAs were first discovered in 1993 and 2000 as key post-transcriptional modulators for the developmental transitions in early larval Caenorhabditis elegans (Banerjee and Slack 2002). Thereafter, an emerging body of experimental evidence substantiated that miRNAs are potential key regulators for diverse developmental and physiological processes such as C. elegans lsy-6 determining

Reprint requests to: Stanley NG Kwang Loong, Bioinformatics Institute, 30 Biopolis Street, \#07-01, Matrix, Singapore 138671; e-mail: stanley@bii.a-star.edu.sg; fax: +65-6478-9050.

Article published online ahead of print. Article and publication date are at http://www.rnajournal.org/cgi/doi/10.1261/rna.223807. the left-right asymmetry of chemo-receptor expression (Johnston and Hobert 2003); Drosophila melanogaster miR-14 miRNA being involved in apoptosis, stress resistance, and fat metabolism (Xu et al. 2003); D. melanogaster bantam repressing the gene hid associated with apoptosis and proliferation (Brennecke et al. 2003); Mus musculus miR-181a modulating hematopoietic differentiation (Chen et al. 2004); M. musculus miR-196 inducing directed cleaving of Hox-B8 transcripts (Yekta et al. 2004); Arabidopsis thaliana miRNAs regulating the expression of transcription factor genes ( $\mathrm{Li}$ and Zhang 2005); and viralencoded miRNAs hijacking the host immune defense to sustain their viral replication and pathogenesis (Grey et al. 2005; Pfeffer et al. 2004, 2005; Samols et al. 2005). This dynamic range of biological findings underscores the functional importance of miRNAs and the need for expanding our limited knowledge concerning them.

The emerging model of miRNA maturation involves six (five) compartmentalized steps in vertebrates (plants) (Anthony and Peter 2005; Kim 2005). Briefly, (1) the 
majority of the primary miRNAs (pri-miRs) are transcribed by RNA polymerase II (Pol-II) into long primary transcripts from the polycistronic genes residing in the intergenic regions that overlap with the introns of protein-coding genes (Lee et al. 2002) or from the exons of the pseudoncRNA genes (Rodriguez et al. 2004). (2) These capped and polyadenylated pri-miRs are processed in the nucleus by an endonuclease RNase III Drosha/Pasha complex yielding $\sim 60-120$-nt intermediate precursor transcripts (pre-miRs) in vertebrates. Conversely, Dicer-like 1 enzyme (DCL1; a plant ortholog of Drosha) performs two cleavage steps in the nucleus: plant pri-miRs $\rightarrow \sim 80-200$-nt pre-miRs $\rightarrow$ $m i R: m i R^{*}$ (Anthony and Peter 2005). (3) Vertebrate and plant pre-miRs possessing characteristic imperfect and extended hairpin structures are exported into the cytoplasm by the Exportin-5 in a Ran-GTP-dependent manner or by HASTY, the ortholog of Exportin-5 (Zhang et al. 2006a). (4) Cytoplasmic RNase III-type endonuclease Dicer excises the vertebrate pre-miR into $\sim 22-23$-nt asymmetric mature miRNA duplex $m i R: m i R^{\star}$. Plant $m i R: m i R^{\star}$ contains more base-pairings and has tighter length distribution centering on $21 \mathrm{nt}$ (Anthony and Peter 2005). (5) The strand $m i R$ with the less thermostable $5^{\prime}$ termini is favorably incorporated into a ribonucleoprotein to form an RNA-induced silencing complex (RISC) (Cullen 2004; Tijsterman and Plasterk 2004; Tang 2005). (6) The RISC represses post-transcriptionally the expression of the targeted gene by translational arrest of protein synthesis via imperfect complementarity at the 3 '-untranslated regions (Moss et al. 1997; Reinhart et al. 2000; Doench and Sharp 2004) or mRNA cleavage degradation with near-perfect complementarity of $\leq 3$ mismatches at the protein-coding regions of mRNAs (Yekta et al. 2004) primarily in vertebrates and plants, respectively (Anthony and Peter 2005).

\section{Existing approaches for identifying pre-miRs or miRNA genes}

Two broad strategies for identifying systematically novel miRNAs exist, in vivo and in silico screening (Ambros et al. 2003; Berezikov et al. 2006). The former, based on expression screening, commences with the isolation of distinct $\sim 22$-nt RNA transcripts. This is followed by intensive direct cloning and sequencing efforts of cDNA libraries derived from the size-fractionated small RNAs (LagosQuintana et al. 2001, 2002; Lau et al. 2001; Lee and Ambros 2001). Such experimental routes are neither exhaustive nor straightforward in discovering all the known miRNAs. Notably, not all miRNAs are well expressed in tissues, cell types, and developmental stages that have been sampled (Lagos-Quintana et al. 2001). Existing cloning methods are highly biased toward abundantly and/or ubiquitously expressed miRNAs that usually dominate the cloned products, rendering the isolation of novel miRNAs difficult (Lagos-Quintana et al. 2001, 2002; Lau et al. 2001; Lee and
Ambros 2001). Novel miRNAs tend to be elusive, as they are expressed constitutively in low abundance or they have preferentially restrictive/specific temporal (cell-phase) and spatial (tissue-/cell-type) expression patterns. To express them sufficiently for cloning efforts under controlled cellular conditions and nonabundant cell types is technically involved. In principle, this issue can be overcome by running high-throughput deep-sequencing technology like massively parallel signature sequencing (MPSS) (Brenner et al. 2000) on appropriately pooled biological samples.

Computational strategies have been applied to C. elegans (Grad et al. 2003; Lim et al. 2003b), D. melanogaster (Lai et al. 2003), A. thaliana, Oryza sativa (Bonnet et al. 2004a; Jones-Rhoades and Bartel 2004; Wang et al. 2004; Adai et al. 2005), Homo sapiens (Berezikov et al. 2005, 2006; Lim et al. 2003a), and viruses (Pfeffer et al. 2004, 2005; Grey et al. 2005; Samols et al. 2005) for identifying candidate miRNAs. In part, they were extensively developed to overcome technical hurdles faced experimentally (Berezikov et al. 2006; Zhang et al. 2006a). Particularly, breakdown products of mRNA transcripts in the background and endogenous ncRNAs (e.g., tRNAs and rRNAs) as well as exogenous siRNAs are dominant players coexisting in the small RNA samples isolated from the cytoplasmic total RNA extracts. To thwart designating these fragments erroneously as novel miRNAs, cloned small RNAs are assessed computationally to identify their genomic location (Lai et al. 2003; Lim et al. 2003a,b; Adai et al. 2005; Fu et al. 2005; Cummins et al. 2006; Wheeler et al. 2006). A critical and necessary feature for mature miRNAs biogenesis is that they reside primarily on one arm of the pre-miRs that form characteristic imperfect hairpin structures. This criterion indicates that only those small RNA sequences occupying the $\sim 20$-nt matched regions on one arm of the hairpin precursors should be curated as novel miRNAs after experimentally validating them. The short sequence length of miRNAs, however, confers relatively low specificity whereby matching regions are readily encoded in an overwhelming number of unwanted genomic segments that can potentially fold into hairpin structures. Genome-wide screening for novel pre-miRs is technically complicated, considering that the hairpin structures are not unique to miRNAs exclusively. These dysfunctional inverted repeats (or pseudohairpins) are genomically prevalent in $H$. sapiens $\left(1.1 \times 10^{7}\right)$ (Bentwich et al. 2005) and C. elegans $\left(4.4 \times 10^{4}\right)$ (Pervouchine et al. 2003) genomes; only 462 and 114 bona fide pre-miRs, respectively, have been discovered according to miRBase 8.2 (Griffiths-Jones et al. 2006).

The majority of the pseudohairpins can be removed by comparative genomic techniques like MiRscan (Lim et al. 2003a,b), MIRcheck (Jones-Rhoades and Bartel 2004), miRFinder (Bonnet et al. 2004a), miRseeker (Lai et al. 2003), findMiRNA (Adai et al. 2005), PalGrade (Bentwich et al. 2005), and MiRAlign (Wang et al. 2005). Typically, conserved regions are first identified by aligning the entire 
genome of phylogenetically related species and masking out those regions most unlikely to be occupied by miRNAs (e.g., tRNAs and rRNAs). Sliding windows of the unmasked regions are folded at both strands by Mfold (Zuker 2003) or RNAfold (Hofacker 2003), two commonly used RNA secondary structure predictors. The folds are scored according to their minimum free energy of folding (MFE), length of the symmetric/asymmetric regions, and size of the terminal loop. The composite scores are thresholded, and those high-ranking ones deemed similar to pre-miRs published in miRBase (Griffiths-Jones et al. 2006) are then reserved for further experimental validation. Evidently, extensive genomics data sets for computationally intensive multiple genome alignments are involved, rendering identification of miRNAs impossible, especially for organisms whose closest relatives have partial or yet-to-start sequenced genomes. Furthermore, species-specific pre-miRs encoded in pathogenic viruses such as the Kaposi sarcoma-associated herpesvirus, Mouse gammaherpesvirus 68, and Human cytomegalovirus are likely to remain elusive to comparative-based detection, as they share little or no sequence homologies among themselves or with the host pre-miRs (Grey et al. 2005; Pfeffer et al. 2004, 2005; Samols et al. 2005).

Several (quasi) de novo state-of-the-art predictors have been extensively developed to aid the discovery of nonconserved pre-miRs and to surmount the technical drawbacks of comparative approaches. Typically, they first decompose the individual pre-miR into modularized RNA substructures comprising dangling termini, asymmetric or symmetric stem, and terminal loop. Derived from these specific regions is a complex array of sequences (e.g., nucleotide composition) and structural characteristics (e.g., thermodynamic stability). This is fashioned analogously to the protein-coding gene identification techniques that scan the genomic regions for signature signals of protein-coding genes without relying on external transcripts or genomic sequences. A supervised machine-learning classification algorithm, e.g., support vector machine (SVM), is trained on a binary-labeled positive set of genuine pre-miRs and a negative set of pseudohairpins. Through this inductive learning on their feature vectors, a classifier model and a set of decision rules are devised to discriminate between them.

An inaugural and definitive work (Pfeffer et al. 2005; Sewer et al. 2005) compiled 40 distinctive sequence and structural features from the hairpins without relying on comparative genomics information-stem length, length of the longest symmetrical region, number of complementary base pairs (bp) in the "relaxed symmetry" region, MFE, number of nucleotides in symmetrical and asymmetrical loops in the "relaxed symmetry" region, and the average size of the asymmetrical loops. The SVM classifier model trained with the experimental domain knowledge recovered $71.00 \%$ of the positive pre-miRs with a remarkably low false-positive rate of $\sim 3.00 \%$. The accuracy was improved to $\sim 90.00 \%$ in human and up to $90.00 \%$ for other species by another de novo classifier, Triplet-SVM (Xue et al. 2005). It encoded the local contiguous structure-sequence features of known pre-miRs as a set of 32 triplet elements-a nucleotide type and three continuous substructures, e.g., "A(((" and "G(...". Despite its methodological simplicity, promising performances, and independence of comparative genomics information, Triplet-SVM was largely limited to classifying RNA secondary structures not containing multiple loops. Another SVM-based approach, RNAmicro (Hertel and Stadler 2006), incorporating sequence and structural information as part of its feature vector, reported incredibly promising efficiencies of $91.16 \%$ and $99.47 \%$ for sensitivity and specificity, respectively. Still, its classification pipeline required computationally expensive multiple sequence alignments for inputs. ProMiR (Nam et al. 2005) took advantage of a probabilistic co-learning model, the hidden Markov model (HMM), to classify miRNA genes based on their pair-wise aligned sequences. It minimized the false-positive rate to as low as $4.00 \%$, but compromised for a poorer performing sensitivity of only $73.00 \%$. A relatively recent work, BayesMIRfinder (Yousef et al. 2006), adopted naive Bayesian induction (NBI) as its underlying classifier model. Notwithstanding its technical novelty, BayesMIRfinder relied on the comparative analysis of conserved genomics regions for postprocessing to yield a considerably higher sensitivity of $97.00 \%$ and comparable specificity of $91.00 \%$ in mouse to existing algorithms.

\section{Motivation and overview of study}

Generally, the efficiency and reliability of classifiers for distinguishing species-specific and evolutionary wellconserved pre-miRs from genomic pseudohairpins and most types of ncRNAs depend largely on the size and selection of both the specific features and the relevant data samples. Existing (quasi) de novo attempts are still limited to and have far from satisfactory predictive performances, hampered largely by the difficulties of deriving and selecting appropriate features from pre-miRs. Proper feature selection should facilitate a more controllable generalization and scalability to new testing samples as well as provide more robust predictive ability to the underlying machine-learning algorithms.

To develop a true de novo predictor that can achieve highly accurate identification and classification of promising precursor transcripts as putative pre-miRs within a single genome, wholly independent of phylogenetic conservation, still entails numerous unforeseeable technical issues. Most notable of these is the previous lack of data and inconclusive findings from existing literature on the features that distinctively distinguish pre-miRs from pseudohairpins and other types of ncRNAs. Motivated by this incomplete knowledge and the many miRNAs present in 
the genomes of vertebrates, worms, plants, and even viruses not yet discovered, we conduct a large-scale characterization study. It comprehensively comprises a heterogeneous collection of 2241 published and nonredundant pre-miRs across 41 species (miRBase 8.2), 8494 pseudohairpins extracted from the human RefSeq genes, 12,387 nonredundant ncRNAs spanning 457 types (Rfam 7.0), 31 full-length mRNAs randomly selected from GenBank, and four sets of synthetically generated genomic background corresponding to each of the native RNA sequence. Hairpin is a crucial structural prerequisite for the computational identification of pre-miR, as its formation is critically associated with the early stages of the mature miRNA biogenesis. To elucidate the unique hairpin folding of an entire pre-miR, our in-depth statistical study focuses solely on their intrinsic and global features at the sequence, structural, and topological levels. The combinatoric features include $\% G+C$ content, normalized base-pairing propensity $P(S)$, normalized minimum free energy of folding $M F E(\mathbf{s})$, normalized Shannon entropy $Q(\mathbf{s})$, normalized base-pair distance $D(\mathbf{s})$, and degree of compactness $F(S)$, as well as their corresponding $Z$ scores of $P(S), \operatorname{MFE}(\mathbf{s}), Q(\mathbf{s})$, $D(\mathbf{s})$, and $F(S)$. The findings will facilitate and promote more accurate guidelines and distinctive criteria for the prediction of authentic pre-miRs with improved performances.

\section{RESULTS AND DICUSSION}

Among the arthropoda, nematoda, vertebrata, viridiplantae, and viruses available from miRBase 8.2 (Griffiths-Jones et al. 2006), no orthologous miRNA gene shared by vertebrates and plants has ever been reported (Anthony and Peter 2005). Pathogenic viral-encoded pre-miRs present in K. sarcoma-associated virus, M. $\gamma$-herpesvirus 68, and H. cytomegalovirus should be treated as exceptions, although they have also been demonstrated to share significant sequence homology neither with known host pre-miRs nor among themselves (Grey et al. 2005; Pfeffer et al. 2005; Samols et al. 2005). Viral-encoded pre-miRs do not possess genes homologous to host miRNA processing proteins, e.g., Drosha, Dicer, and RISC, but are likely to hijack these proteins to facilitate their viral replication after infecting the host cells (Sarnow et al. 2006). Despite the apparent similarities of miRNA biogenesis between vertebrates and plants, their evolutionarily ancient processing pathways ( $\geq 400$ million years ago) were not operating in a common ancestor and could have evolved independently from a more ancient system (Anthony and Peter 2005). We will focus on vertebrate and plant pre-miRs for discussion, as they are likely to exhibit distinct folding features that warrant careful structural analysis. Data are available to deduce conclusions about arthropoda, nematoda, and virus pre-miRs. (Supplemental Materials can be found at http:// web.bii.a-star.edu.sg/ stanley/Publications.)

\section{Vertebrate and plant pre-miRs have significantly distinct $M F E I_{2}, M F E I_{1}, \% G+C, P(S), M F E(s), Q(s)$, $D(\mathrm{~s})$, and $F(S)$ from ncRNAs and mRNAs}

Foremost, the sequence length (in nucleotides) differs considerably between and among pre-miRs (vertebrate, $90.4522 \pm$ 0.4164 and plants, $137.9175 \pm 2.0309$ ), ncRNAs (frameshift, $53.2599 \pm 0.2543$ to IRES, $276.0841 \pm 2.4342$ ), and mRNA (332.3226 \pm 16.3064$)$ (Fig. 1A, Figure 3A, top heat map, see below; Supplemental Table S1). The sequence lengths of ncRNAs and mRNAs are strongly and positively correlated with their MFEs, as previously demonstrated (Seffens and Digby 1999; Bonnet et al. 2004b; Zhang et al. $2006 \mathrm{~b})$. Longer sequence length results in a greater degree of freedom such that the native RNA sequences can fold into complex secondary structures with corresponding higher thermostability or lower MFEs. By normalizing the MFE with the sequence length, the normalized MFE, $M F E(\mathbf{s})$, ensures that it serves as a comparable measure without unduly penalizing the shorter pre-miRs or favoring the longer mRNAs (Seffens and Digby 1999; Freyhult et al. 2005; Zhang et al. 2006b). In agreement with earlier findings (Freyhult et al. 2005; Zhang et al. 2006b), vertebrate and plant pre-miRs possess statistically distinct $\operatorname{MFE}(\mathrm{s})$ of $-0.4308 \pm 0.0025$ and $-0.4456 \pm 0.0038(p<$ $0.001)$ and are the lowest except frameshift $(-0.4814 \pm$ $0.0023)$. Interestingly, a single criterion based on a variant of $\operatorname{MFE}(\mathbf{s})$ greater than a threshold value $\varepsilon=0.68$ has been applied to genome-wide detection of $C$. elegans premiRs (Pervouchine et al. 2003). This yielded $\sim 4.4 \times 10^{4}$ stable hairpins localized to $\sim 4.00 \%$ of the genome, covering 64.29\% (36/56) of the published ones (Lau et al. 2001).

Vertebrate and plant pre-miRs possess the significantly highest normalized base-pairing propensity $P(S)$ of $0.3518 \pm$ 0.0009 and $0.3545 \pm 0.0013(p<0.001)$, accounting for $\sim 70.36-70.9 \%$ of their nucleotides forming complementary base pairings within their highly thermostable hairpin structures. A similar $>72.00 \%$ for $P(S)$ has also been reported, corroborating our findings, albeit a smaller data set of 513 plant pre-miRs across seven species was analyzed (Zhang et al. 2006b). The presence of more hydrogen bonds and base-pairings in the plant pre-miRs might benefit their recognition, processing, and nucleus-cytoplasm transport (Zhang et al. 2006b). Emerging experimental evidence also points to the hairpin motif of vertebrate pre-miRs as a critical feature for miRNA maturation (Zeng and Cullen 2004). Human pre-miR-30 binding by Exportin-5 involved recognition of almost the entire hairpin, except the terminal loop (Zeng and Cullen 2004). A hairpin structure $>16$ bp was required for detectable binding and $>18$ bp for high-affinity binding such that the stacking of contiguous paired nucleotides tended to reduce the MFE of the overall folded structure for greater thermostability. Contrary to the common belief that the unpaired regions tended to disrupt the RNA structure with greater MFE, deleting the 2-nt bulge of 


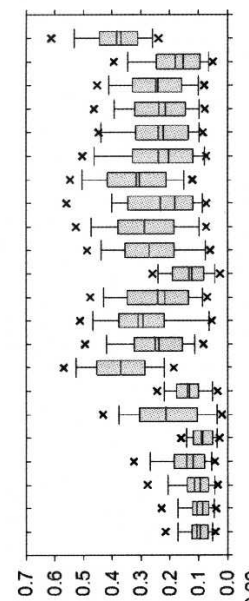

(s) 0

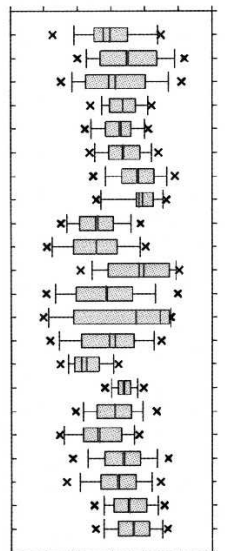

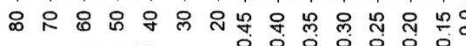
(s)d

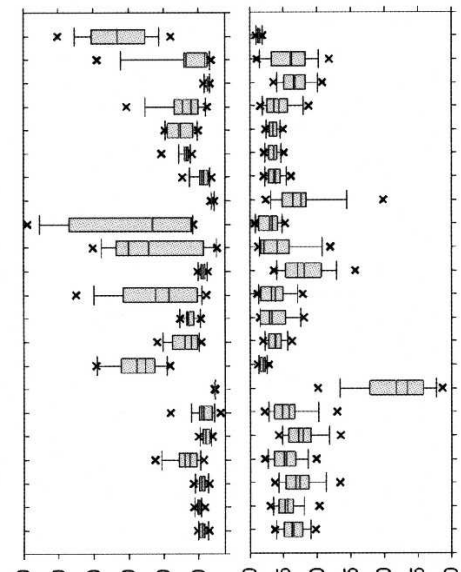

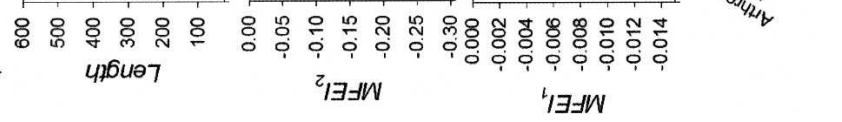

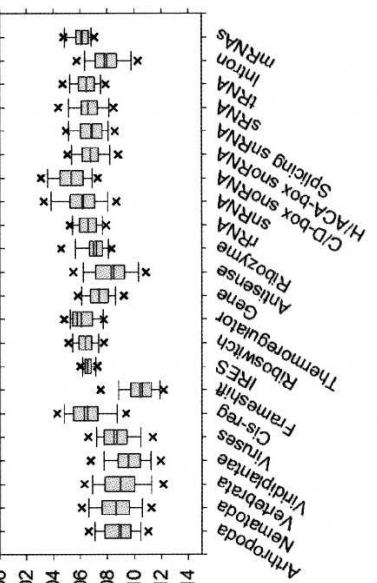

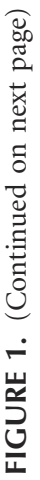




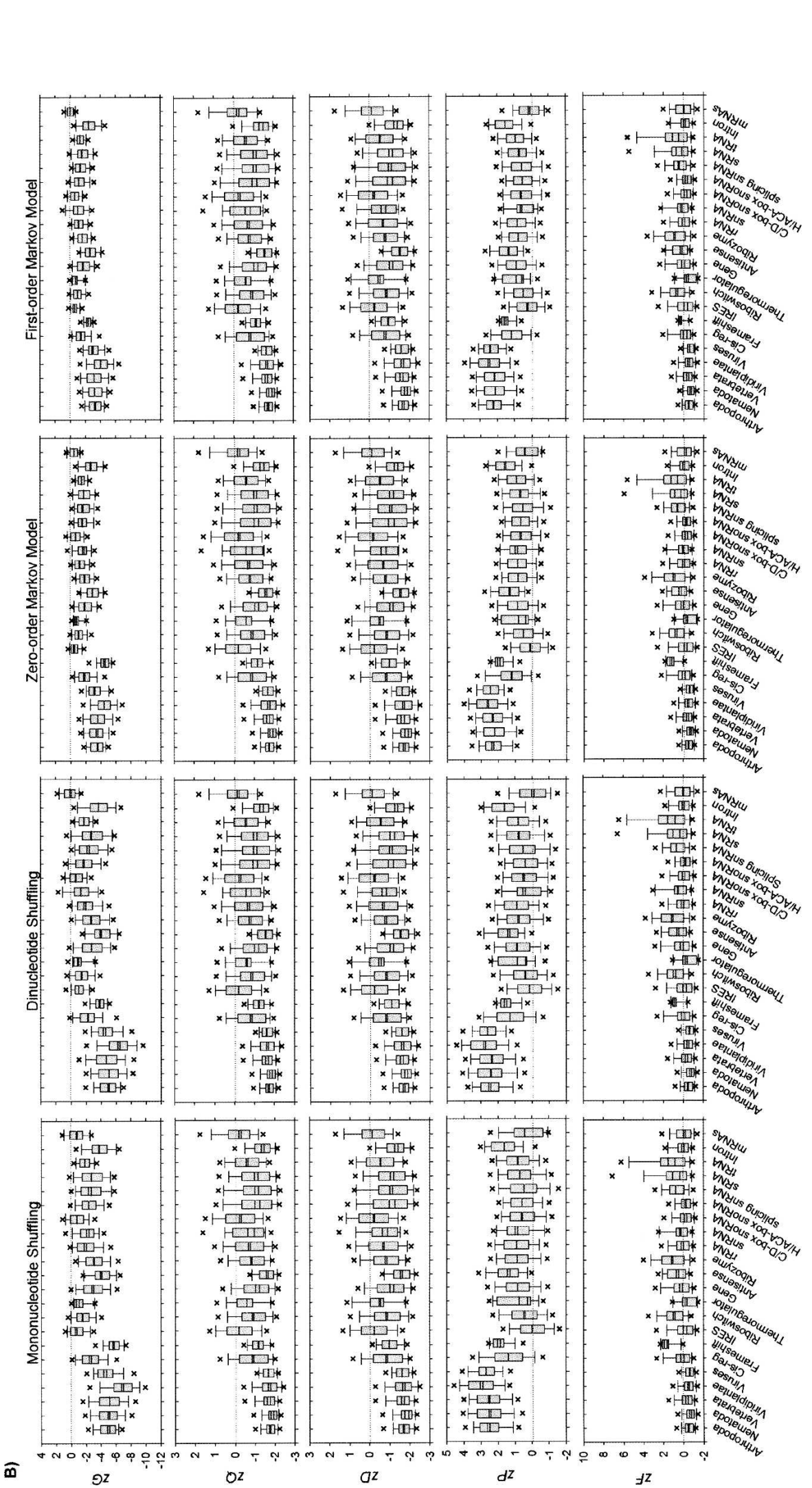

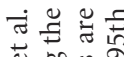

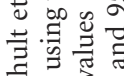

矛额矛

这芯. 莺

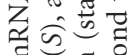

घ $\approx$ च ठ

क त

जิ

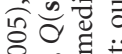

की

坞素

峲

을 㫕

웡은

可 $\Xi$

记

䓀.

ध

古运

范资

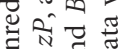

등

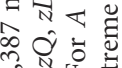

जि.

ลิ요웜

तें

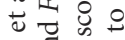

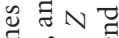

우욯

击会选

苛

$\hat{\omega} \cdot \Xi \frac{\pi}{.0}$

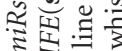

रें

늠 吾

䒿

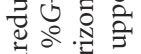

공회을

स्丶

ปิ

它志的

धै क्षेत्र

든 흔

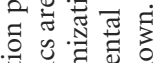

.

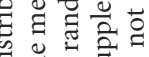

ดิ

등

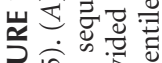

은 令 
pre-miR-30 left the binding unaffected or reduced binding modestly, unless the stem length was suboptimal. There was negligible or no significant effect on the correct recognition for varying sizes of the terminal loop, until it was shortened from the normal 15-4 nt. Besides nuclear export of pre-miR, the binding of Exportin-5 served to stabilize the pre-miR in the nucleus and during export by inhibiting the in vitro exonucleolytic cleavage (Zeng and Cullen 2004).

Vertebrate and plant pre-miRs encode higher $\% A+U$ content than $\% G+C$ content of $48.3079 \pm 0.2504$ and $46.6719 \pm 0.3513$; similarly observed (Zhang et al. 2006b). The higher $\% A+U$ content in the plant pre-miRs (likewise for vertebrate pre-miRs) might possibly serve as a biochemical signal for miRNA biogenesis by the RISC (Zhang et al. 2006b). We also report that the $\% G+C$ contents for vertebrate and plant pre-miRs are not considerably different from mRNAs $(50.4626 \pm 1.4654)$ and common families of ncRNAs like cis-regulator (48.9672 \pm 0.1188$)$, frameshift (46.4785 \pm 0.1477$)$, riboswitch $(50.5054 \pm 0.3381)$, thermoregulator (42.6490 \pm 3.2009$)$, HACA-box snoRNA (46.3048 \pm 0.3160), splicing RNA (47.6933 \pm 0.3731), sRNA (46.3963 \pm 0.3513$),$ tRNA (48.2725 \pm 0.3541$)$, and intron (44.7871 \pm 0.8350$)$. Unlike the $\% G+C$ content, the $\mathrm{MFEI}_{1}$ [divides $\mathrm{MFE}(\mathbf{s})$ by $\% G+C$ content, a newly proposed folding energy score to analyze plant pre-miRs; Zhang et al. 2006b] for vertebrate and plant pre-miRs of $-0.0091 \pm$ 0.0001 and $-0.0096 \pm 0.0001$ are statistically highest $(p<$ $0.001)$ except anti-sense $(-0.0083 \pm 0.0001)$ and frameshift $(-0.0104 \pm 0.0000)$. Our finding and another (Zhang et al. 2006b) point to the $M F E I_{1}$ as a potential discriminative criterion to distinguish pre-miRs from mRNAs and ncRNAs, which a recent comparative classifier RNAmicro has included into its feature set (Hertel and Stadler 2006).

Notably, vertebrate pre-miRs possess statistically higher normalized Shannon entropy $Q(\mathbf{s})$ and normalized basepair distance $D(\mathbf{s})$ of $0.1161 \pm 0.0025$ and $0.0431 \pm 0.0009$ than plant pre-miRs of $0.1424 \pm 0.0036$ and $0.0502 \pm$ $0.0011(p<0.001)$. Generally, RNA sequences having relatively high values of both advanced folding measures are either unstructured or long in length, which fold with the assistance of accessory proteins or have a repertoire of alternative (pseudoknot) structures (Freyhult et al. 2005). This suggests that vertebrate pre-miRs will likely fold into well-defined hairpins restricted to relatively fewer alternative conformations, possibly due to shorter sequence length $(90.4522 \pm 0.4164 \mathrm{nt})$ compared to plants (137.9175 \pm $2.0309 \mathrm{nt})$. The different "structureness" of vertebrate and plant pre-miRs causes the former to display the significantly lowest and distinct $Q(\mathbf{s})$ and $D(\mathbf{s})(p<0.001)$ except anti-sense $(0.1336 \pm 0.0061$ and $0.0468 \pm 0.0020)$. The latter is not significantly unique from cis-regulator $(0.2124 \pm$ 0.0021 and $0.0689 \pm 0.0006)$, frameshift $(0.1396 \pm 0.0024$ and $0.0552 \pm 0.0009)$, anti-sense $(0.1336 \pm 0.0061$ and $0.0468 \pm .0020)$, snRNA $(0.2305 \pm 0.0260$ and $0.0741 \pm$ $0.0074)$, and intron $(0.1802 \pm 0.0089$ and $0.0620 \pm 0.0026)$.
Maturation of the plant $m i R: m i R^{\star}$ duplex is performed exclusively by Dicer-like 1 enzyme (DCL1) via two cleavage steps, pri-miR $\rightarrow$ pre-miR $\rightarrow m i R: m i R^{\star}$, within the nucleus. In contrast to vertebrates (Anthony and Peter 2005; Zhang et al. 2006a), the two reactions are compartmentalized and directed separately by the nuclear Drosha $($ pri-miR $\rightarrow$ pre$m i R)$ and cytoplasmic Dicer $\left(p r e-m i R \rightarrow m i R: m i R^{*}\right)$. Moreover, plant pre-miRs are less conserved (conservation of plant mature miRNAs is well preserved) than those in vertebrates (Anthony and Peter 2005; Zhang et al. 2006a). Our structural analysis substantiates both experimental findings, pointing to the plant pre-miRs as very transient molecules (Zhang et al. 2006a) that possess less "structureness" indicative of lower $Q(\mathbf{s})$ and $D(\mathbf{s})$ compared to their vertebrate counterparts.

Finally, we analyzed two newly proposed topological measures, i.e., degree of compactness $F(S)$ and $\mathrm{MFEI}_{2}$ [divides $M F E(\mathbf{s})$ by number of stems $S$ ]. Vertebrate premiRs have a significantly higher $F(S)$ of $0.2197 \pm 0.0042$ than plant pre-miRs of $0.1251 \pm 0.0033(p<0.001)$. Generally, RNAs possessing lower $F(S)$ have less structured folds (Barash 2003, 2004) like mRNAs (0.0391 \pm 0.0059). Both vertebrate and plant pre-miRs fold into topologically distinct structures with $F(S)$ being statistically different $(p<$ $0.001)$ but not the extreme among mRNAs (0.0391 \pm $0.0059)$ and common families of ncRNAs like frameshift $(0.8865 \pm 0.0079)$, IRES (0.0442 \pm 0.0013), anti-sense $(0.3734 \pm 0.0133)$, rRNA (0.0933 \pm 0.0020$)$, snRNA $(0.5372 \pm 0.0415)$, and tRNA $(0.5333 \pm 0.0093)$. The other folding measure, $M F E I_{2}$, was inspired by the formation of the critical hairpin structure in the early stages of miRNA maturation. Reasonably, MFE should be largely localized to the stem(s) within the hairpin such that the higher $\mathrm{MFEI}_{2}$ corresponds to greater thermostability per stem. The $\mathrm{MFEI}_{2}$ for vertebrate and plant pre-miRs of $-0.0761 \pm 0.0013$ and $-0.0539 \pm 0.0010$ are significantly different $(p<0.001)$ except anti-sense $(-0.0811 \pm 0.0030)$, snRNA $(-0.0764 \pm 0.0088)$, and tRNA $(-0.0676 \pm$ $0.0007)$, cis-regulator $(-0.0793 \pm 0.0017)$, snRNA $(-0.0764 \pm 0.0088)$, and intron $(-0.0604 \pm 0.0029)$.

In summary, the 1203 vertebrate and 606 plant pre-miRs are statistically distinct from 12,387 ncRNAs and 31 mRNAs according to the measures $M F E I_{2}, M F E I_{1}, \% G+C, P(S)$, $\operatorname{MFE}(\mathbf{s}), Q(\mathbf{s}), D(\mathbf{s})$, and $F(S)$. Except two recent published works investigating 513 plant pre-miRs (Zhang et al. 2006b) and 135 pre-miRs from different species (Freyhult et al. 2005), we are unaware of any larger-scale and in-depth statistical analysis highlighting these results on the folding characteristics of published pre-miRs.

\section{Vertebrate and plant pre-miRs have significantly distinct $Z$ scores of $M F E(s), Q(s), D(s), P(S)$, and $F(S)$ compared to the ncRNAs and mRNAs}

Evolutionarily conserved vertebrate and plant pre-miRs possess the considerably lowest $z G(p<0.001)$ except frameshift 
and anti-sense, regardless of the sequence randomization algorithms (Fig. 1B, Figure 3A, bottom heat map, see below; Supplemental Table S2). Our finding and another (Freyhult et al. 2005) affirm the hypothesis that pre-miRs fold into highly thermostable secondary structures with significantly lower MFEs relative to their synthetically generated sequence randomized controls (Workman and Krogh 1999; Bonnet et al. 2004b). Therefore this unique structural characteristic of vertebrate and plant pre-miRs is not expected to occur by chance; it is indispensable for correct recognition and processing by Dicer-like enzymes (Bonnet et al. 2004b). Earlier works (Workman and Krogh 1999; Bonnet et al. 2004b) were inconclusive, as their dinucleotide shuffling algorithms were heuristically based, and the resulting shuffled RNAs might not guarantee preserving the exact dinucleotide frequencies as the native RNAs (Clote et al. 2005). Instead, we used a considerably larger data set of pre-miRs and ncRNAs as well as the exact "Altschul-Erickson algorithm" (Altschul and Erickson 1985) for synthesizing $10^{4}$ dinucleotide-shuffled RNAs. Two computational studies (Washietl and Hofacker 2004; Clote et al. 2005) also demonstrated that structural ncRNAs displayed lower MFEs than dinucleotide-shuffled RNAs, but pre-miRs were not analyzed.

Both $z Q$ and $z D$ of vertebrate and plant pre-miRs are statistically different $(p<0.001)$ and are the lowest except anti-sense, irrespective of the sequence randomization algorithms. A recent computational study reported that pre-miRs and ncRNAs (like hammerhead ribozyme type III and tRNAs) possessed significantly fewer $k$-locally optimal structures (potential kinetic traps) than their dinucleotide-shuffled RNAs (Clote 2005). Both findings suggest pre-miRs are likely to undergo evolutionary pressure in adopting relatively fewer alternative folds of significantly lower MFEs than the random background, in order to function properly in the post-transcriptional gene regulatory pathway.

Vertebrate and plant pre-miRs report the significantly highest $z P(p<0.001)$; i.e., more complementary basepairings are present in their RNA secondary structures than the genomic background, irrespective of the sequence randomization methods. They also have statistically distinct $z F(p<0.001)$ except common families of ncRNAs like cis-regulator, IRES, thermoregulator, CD-box snoRNA, and HACA-box snoRNA, as well as mRNAs.

In summary, the 1203 vertebrate and 606 plant pre-miRs are significantly different from the 12,387 ncRNAs and 31 mRNAs, after examining their $z G, z Q, z D, z P$, and $z F$ based on four sequence randomization algorithms and $10^{4}$ random sequences corresponding to each native RNA sequence. This statistical finding confirms that to reliably identify pre-miRs from the genomic background requires more than their possessing characteristic and well-defined secondary structures of statistically significant MFEs (Rivas and Eddy 2000; Washietl and Hofacker 2004).

\section{Comparison with previous studies on structural folding analysis of ncRNAs and mRNAs}

For completeness of this large-scale study, we outline three notable points to revisit previous works investigating whether ncRNAs and mRNAs fold into statistically significant and thermodynamically stable secondary structures (Fig. 1B, Figure 3A, bottom heat map, see below; Supplemental Table S2). First, 51 mRNAs had significantly lower MFEs than their corresponding sets of 10 mononucleotide-shuffled RNAs (Seffens and Digby 1999) and a subset of 46 mRNAs did not display any statistically lower MFEs than their corresponding sets of 10 dinucleotide-shuffled RNAs (Workman and Krogh 1999). Our study (mononucleotide shuffling, $-0.7223 \pm 0.2089$ and dinucleotide shuffling, $0.1021 \pm 0.1625)$ and another using dinucleotide shuffling (Freyhult et al. 2005) support both previous conclusions (Seffens and Digby 1999; Workman and Krogh 1999). Unique to this work, we observe that the mRNAs have considerably lower MFEs than the genomic background for the zero-order Markov model $(-0.4770 \pm 0.1098)$, but not for the first-order Markov model $(-0.0830 \pm 0.0845)$.

Second, our investigated 1114 tRNAs possess significantly lower MFEs than the genomic background for the four sequence randomization methods. This finding agrees with earlier results (Washietl and Hofacker 2004; Clote et al. 2005; Freyhult et al. 2005) that relied on dinucleotide-shuffled RNAs, but differs from another work (Workman and Krogh 1999) in which the dinucleotide-shuffling algorithm was heuristically based, as previously explained (Clote et al. 2005). We report similar findings for hammerhead ribozyme type III (Washietl and Hofacker 2004; Clote et al. 2005), spliceosomal RNAs (Washietl and Hofacker 2004; Clote et al. 2005), riboswitches (Clote et al. 2005), and introns (Washietl and Hofacker 2004) that have considerably lower MFEs than corresponding sets of dinucleotide-shuffled RNA sequences.

Third, previously discussed (Workman and Krogh 1999; Bonnet et al. 2004b; Clote et al. 2005), the controls serving as the genomic background would give erroneous conclusions if they destroyed certain nonrandom compositions of the native sequence. Our results highlight that detectable systematic biases of $z G$ distribution profiles exist among the four sequence randomization algorithms. Generally, the mean $z G$ for pre-miRs, ncRNAs, and mRNAs are ordered from the lowest mononucleotide shuffling, marginally below those of dinucleotide shuffling, followed by the zero- and first-order Markov model. This result agrees with earlier works (Workman and Krogh 1999; Bonnet et al. 2004b; Clote et al. 2005) in which disrupting the naturally occurring biases in the inherent dinucleotide frequencies of the sequences base composition should be avoided for determining the significance of secondary structure. Preserving the dinucleotide frequencies of the native sequences is critical so as not to affect the critical energy contributions of stacked base pairs and the corresponding 
A)
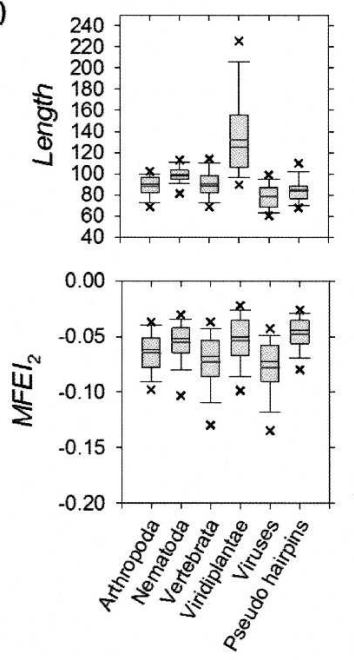

B)
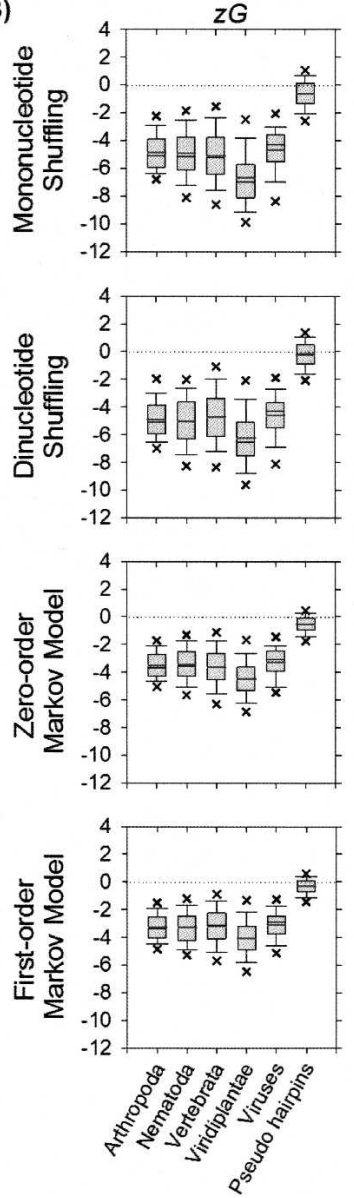
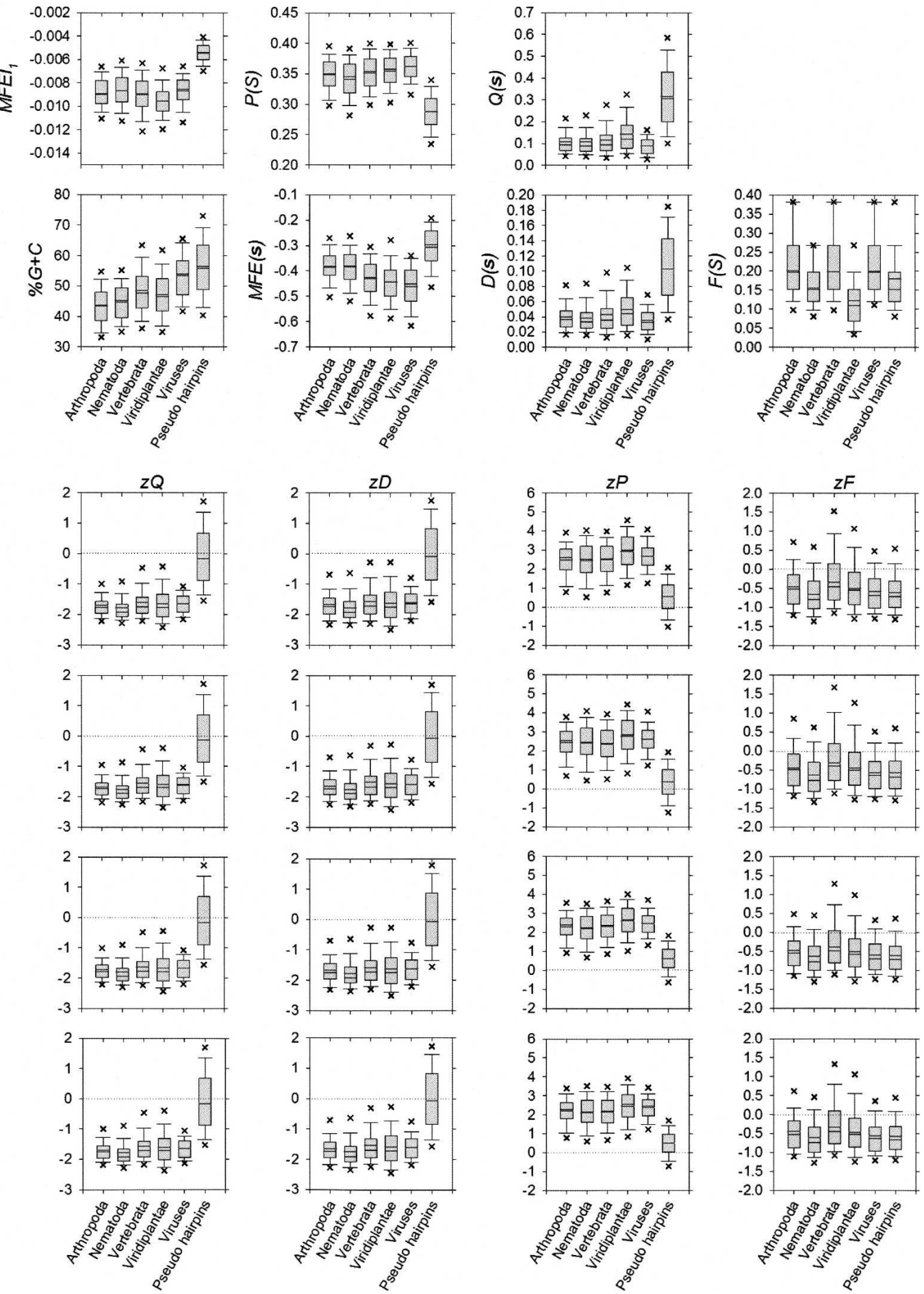

FIGURE 2. Distribution profiles for the 2241 nonredundant pre-miRs (Griffiths-Jones et al. 2006) and 8494 pseudohairpins (Xue et al. 2005). (A) Nine metrics are Length, $M F E I_{2}, M F E I_{1}, \% G+C, P(S), M F E(\mathbf{s}), Q(\mathbf{s}), D(\mathbf{s})$, and $F(S)$. (B) $z G, z Q, z D, z P$, and $z F$, i.e., normalized forms of $M F E(\mathbf{s}), Q(\mathbf{s}), D(\mathbf{s}), P(S)$, and $F(S)$ using the four sequence randomization algorithms. The horizontal dashed line indicates $Z$-score at zero. For $A$ and $B$, box lines indicate the lower quartile, median, mean (statistical values are provided in Supplemental Tables S1 and S2), and upper quartile; whisker lines extend to the most extreme data value or at most 1.5 times the box height; outliers beyond the fifth and 95th percentiles are not shown. 
A)
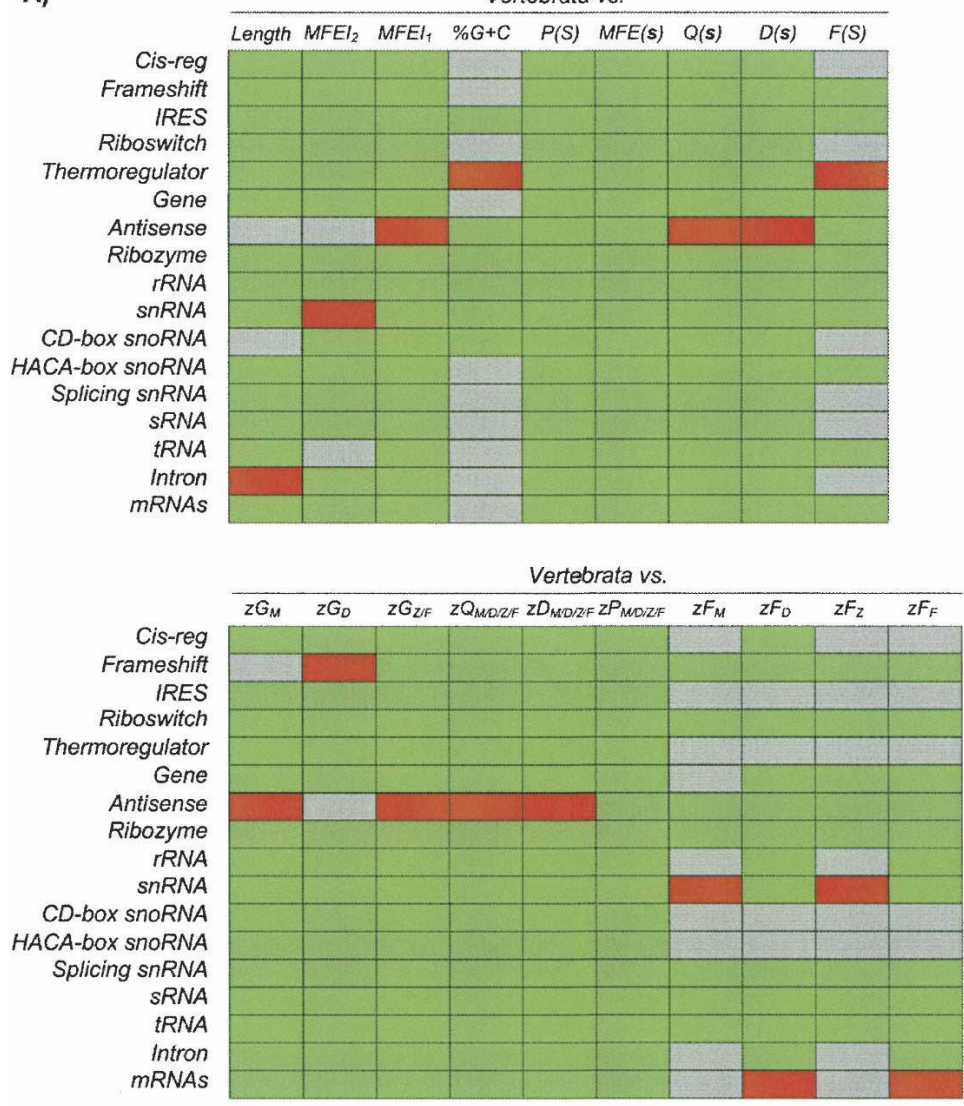

B)
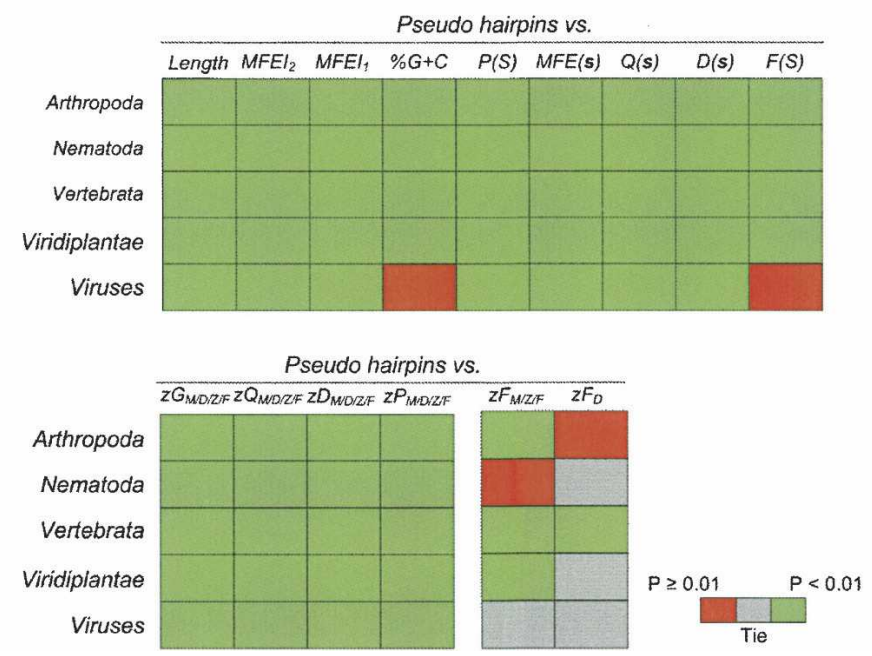
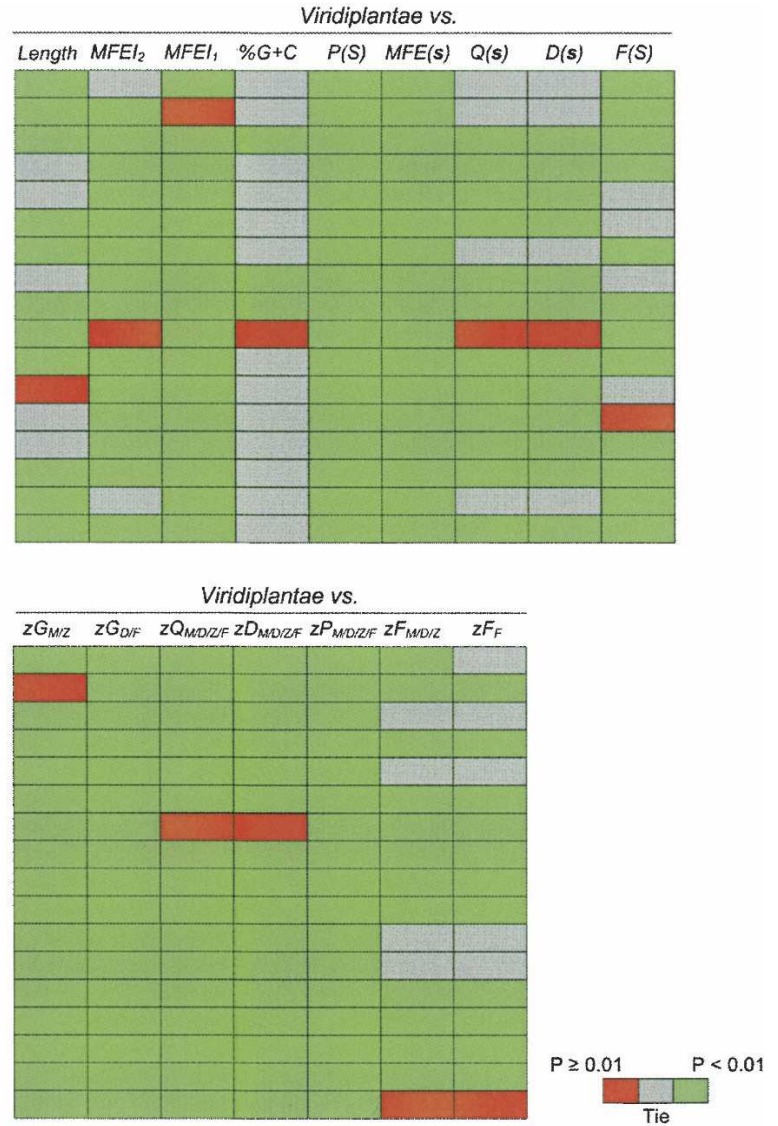

C)
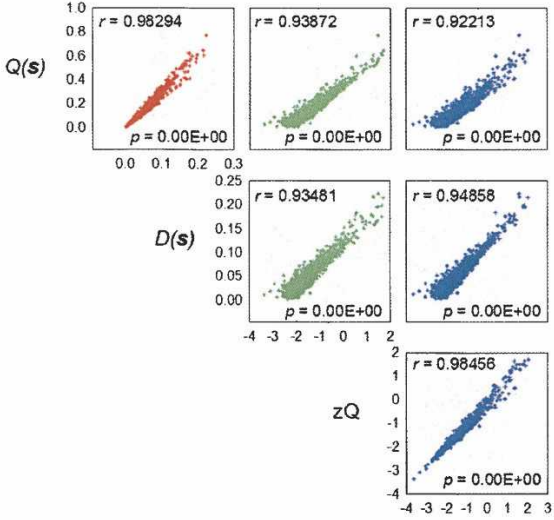

$z D$

FIGURE 3. (A) Heat map of 1203 vertebrate and 606 plants pre-miRs versus 12,387 nonredundant ncRNAs (Griffiths-Jones et al. 2005) and 31 mRNAs (Freyhult et al. 2005). (B) Heat map of 2241 nonredundant pre-miRs (Griffiths-Jones et al. 2006) versus 8494 pseudohairpins (Xue et al. 2005). For $A$ and $B$, e.g., $z G_{M / D / Z / F}$ denotes $z G$ with respect to mono- and d-nucleotide shuffling, zero- and first-order Markov model; green represents statistically different median, red for no statistical difference, gray for ties, according to the nonparametric Kruskal-Wallis one-way ANOVA and Dunn's method of multiple comparisons tests at $p<0.001$ and $p<0.01$. (C) Correlation between $Q(\mathbf{s}), D(\mathbf{s}), z Q$, and $z D$ for 2241 nonredundant pre-miRs; $z Q$, and $z D$ correspond to dinucleotide shuffling; $r$ indicates Pearson correlation coefficients $C_{p}$. The Pearson $C_{p}$, Spearman rank $C_{s}$ (ranks based), and Kendall's $C_{k}$ (relative ranks based) correlation coefficients for all the metrics and sequence randomization methods studied in this work are provided in Supplemental Table S3. 
TABLE 1. Biologically relevant data sets and annotation information

\begin{tabular}{|c|c|c|c|}
\hline Data sets & Counts & Annotation information & Source \\
\hline $\begin{array}{l}\text { Precursor miRNAs } \\
\quad(\text { pre-miRs })^{\mathrm{a}}\end{array}$ & 2241 & $\begin{array}{l}\text { Arthropoda (4/171): Anopheles gambiae, Apis mellifera, } \\
\text { Drosophila melanogaster, Drosophila pseudoobscura } \\
\text { Nematoda (2/189): Caenorhabditis briggsae, Caenorhabditis elegans } \\
\text { Vertebrata (19/1203): Xenopus laevis, Xenopus tropicalis, Gallus gallus, } \\
\text { Canis familiaris, Ateles geoffroyi, Lagothrix lagotricha, Saguinus labiatus, } \\
\text { Macaca mulatta, Homo sapiens, Pan troglodytes, Lemur catta, Mus musculus, } \\
\text { Rattus norvegicus, Bos taurus, Ovis aries, Sus scrofa, Danio rerio, Fugu rubripes, Tetraodon nigroviridis } \\
\text { Viridiplantae (9/606): Arabidopsis thaliana, Glycine max, Medicago truncatula, Oryza sativa, } \\
\text { Physcomitrella patens, Populus trichocarpa, Saccharum officinarum, Sorghum bicolor, Zea mays } \\
\text { Viruses (7/72): Epstein Barr virus, Herpes Simplex Virus 1, Human cytomegalovirus, Kaposi } \\
\text { sarcoma-associated herpesvirus Mouse gammaherpesvirus 68, Rhesus lymphocryptovirus, Simian virus } 40\end{array}$ & $\begin{array}{l}\text { miRBase } 8.2 \\
\text { (Griffiths-Jones } \\
\text { et al. 2006) }\end{array}$ \\
\hline $\begin{array}{l}\text { Noncoding RNAs } \\
(\text { ncRNAs })^{b}\end{array}$ & 12387 & 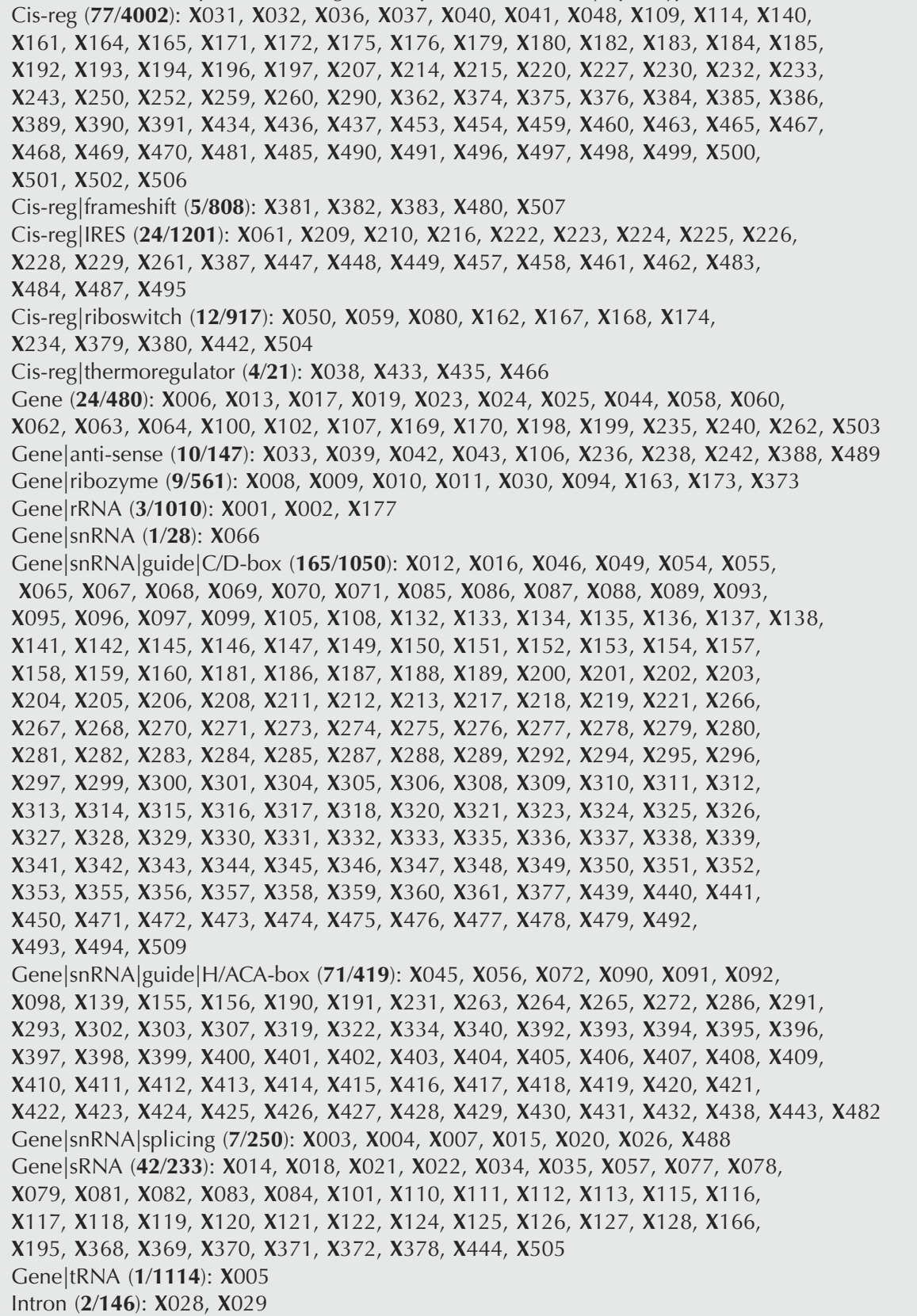 & $\begin{array}{l}\text { Rfam } 7.0 \\
\quad \text { (Griffiths-Jones } \\
\text { et al. 2005) }\end{array}$ \\
\hline
\end{tabular}


TABLE 1. Continued

\begin{tabular}{|c|c|c|c|}
\hline Data sets & Counts & Annotation information & Source \\
\hline mRNAs ${ }^{c}$ & 31 & $\begin{array}{l}\text { NM_001005151.1, NM_001003967.1, NM_177233.4, AY675236.1, } \\
\text { NM_001004202.1, NM_178539.2, AB164385.1, AY555511.1, AB189435.1, } \\
\text { NM_178307.2, NM_001003966.1, NM_205498.1, NM_013564.3, Z81556.1, } \\
\text { NM_131070.2, X56279.1, AK045412.1, AF452886.1, BC049701.1, BC050086.1, } \\
\text { NM_172343.1, AY182163.1, BC072691.1, CV127341.1, NC_004671.1, X00910.1, } \\
\text { AY226143.1, AJ621386, CV122154.1, X68284, and CV199185.1 }\end{array}$ & $\begin{array}{l}\text { GenBank } \\
\quad \text { (Benson et al. 2005) }\end{array}$ \\
\hline Pseudohairpins & 8494 & & $\begin{array}{l}\text { UCSC Browser } \\
\text { (Karolchik et al. 2003) } \\
\text { RefSeq (Pruitt and } \\
\text { Maglott 2001) }\end{array}$ \\
\hline
\end{tabular}

(Counts) Number of sequences being investigated: ${ }^{a}$ For example, phylum Arthropoda (4/171) has four species of pre-miRs containing 171 sequences. ' ${ }^{\mathrm{F}}$ For example, family Cis-reg (77/4002) has 77 types of ncRNAs containing 4002 sequences; miRBase accession X005 abbreviates RF00005. 'GenBank accession numbers. (Source) miRBase Registry Database release 8.2, http://microrna.sanger.ac.uk/sequences; Rfam Database release 7.0, http://www.sanger.ac.uk/Software/Rfam; GenBank DNA Database, http://www.ncbi.nlm.nih.gov/Genbank; UCSC Browser, http://genome.ucsc.edu; RefSeq, http://www.ncbi.nlm.nih.gov/RefSeq.

accuracy of the RNA structural predictions (Workman and Krogh 1999; Bonnet et al. 2004b; Clote et al. 2005).

\section{Vertebrate and plant pre-miRs are significantly different from pseudohairpins}

To elucidate the unique folding of pre-miRs present in vertebrates and plants, we repeat the preceding two statistical experiments by evaluating them against 8494 pseudohairpins instead of ncRNAs and mRNAs. Pseudohairpins are genomic inverted repeats extracted from the proteincoding regions of human RefSeq genes with no known alternative splicing (AS) events. They were first introduced as negative samples in Triplet-SVM (Xue et al. 2005), a de novo classifier based on triplet-encoding features, e.g., "A(((" and "G(..". However, no structural analysis or comparison to published pre-miRs has been reported about them.

Generally, the vertebrate and plant pre-miRs have significantly higher $P(S)$ and $F(S)$ as well as lower $M F E I_{2}, M F E I_{1}$, $\% G+C, M F E(\mathbf{s}), Q(\mathbf{s})$, and $D(\mathbf{s})$ than pseudohairpins $(p<0.001)$ (Fig. 2A; Fig. 3B, top heat map; Supplemental Table S1). The distribution profiles of vertebrate and plant pre-miRs for $z G, z Q, z D$, and $z P$ differ distinctively from pseudohairpins $(p<0.001)$, irrespective of the sequence randomization algorithms (Fig. 2B; Fig. 3B, bottom heat map; Supplemental Table S2). Unlike pseudohairpins, premiRs tend to fold into secondary structures with significantly higher thermodynamic structural stability (lower $z G$ ), fewer alternative folds (lower $z Q$ and $z D$ ), and more base-pairings (higher $z P$ ). Except plants, vertebrate pre-miRs clearly have significantly higher $z F$ (more compactness) than pseudohairpins $(p<0.001)$.

In summary, both findings invalidate conclusively the hypothesis that pseudohairpins share a comparable degree of structural folding characteristics with known vertebrate and plant pre-miRs. Our statistical results clearly point to the $M F E I_{2}, M F E I_{1}, \% G+C, P(S), M F E(\mathbf{s}), Q(\mathbf{s}), D(\mathbf{s})$, and $F(S)$ as well as $z G, z Q, z D, z P$, and $z F$ as potential discriminative descriptors. They effectively expand the triplet-encoding features in Triplet-SVM (Xue et al. 2005) to classify more accurately the genuine pre-miRs from pseudohairpins in genome-wide screens.

\section{Correlation between folding measures}

We conducted correlation tests on 2241 nonredundant known pre-miRs according to the following metrics: Length, $M F E I_{2}, M F E I_{1}, \% G+C, P(S), M F E(\mathbf{s}), Q(\mathbf{s}), D(\mathbf{s})$, and $F(S)$ as well as the $z G, z Q, z D, z P$, and $z F$ [normalized forms of $M F E(\mathbf{s}), Q(\mathbf{s}), D(\mathbf{s}), P(S)$, and $F(S)$ using the four sequence randomization algorithms] (Fig. 3C; Supplemental Table S3). The Pearson correlation coefficients $C_{p}$ are also validated against Spearman rank $C_{s}$ (ranks based) and Kendall's $C_{k}$ (relative ranks based) correlation coefficients, as $C_{s}$ and $C_{k}$ are extremely robust to non-normal distribution.

Generally, all of the metrics are weakly $\left(\left|C_{p}\right|<0.4\right)$ and moderately $\left(0.4<\left|C_{p}\right|<0.9\right)$ correlated except $Q(\mathbf{s}), D(\mathbf{s})$, $z Q$, and $z D$, regardless of the sequence randomization algorithms. Both $Q(\mathbf{s})$ and $D(\mathbf{s})$ are computed from the McCaskill base-pair probability $p_{i j}$ (Freyhult et al. 2005), explaining the strong quasilinear relationship $\left(C_{p} \geq 0.9\right)$ for the two pairs $Q(\mathbf{s})$ and $D(\mathbf{s})$ as well as their corresponding normalized form $z Q$ and $z D$. There exist moderate Pearson correlations within the three pairs $M F E(\mathbf{s})$ and $z G$, $P(S)$, and $z P$, as well as $F(S)$ and $z F$ for the four sequence randomization algorithms. We initially expected $Q(\mathbf{s})$ and $z Q$ as well as $D(\mathbf{s})$ and $z D$ to behave similarly. Interestingly and currently unclear is why a strong association is observed within them. As a guide for future studies, especially where computational resources are limited, only $Q(\mathbf{s})$ instead of $D(\mathbf{s})$ should be included (Freyhult et al. 2005), 
while $z Q$ and $z D$ are extremely time consuming to compute beyond $10^{3}$ random RNA sequences.

\section{CONCLUSIONS}

In this large-scale investigation characterizing the entire hairpin structure of known precursor miRNAs (pre-miRs), we have demonstrated that they (notably, vertebrate and plant pre-miRs) possess a set of 13 statistically significant global features. Our in silico findings have greatly advanced our understanding of miRNA functions and biogenesis in relation to their structural features and distinct folding patterns. A definitive criterion for identifying and classifying accurately promising precursor transcripts as bona fide pre-miRs while discriminating against abundant pseudohairpins within a single genome has not yet been discovered. Moreover, discriminative features used in existing (quasi) de novo classifiers have achieved far from satisfactory specificity and sensitivity, especially when cross-species conservation is unavailable. Our investigated features relating to the intrinsic folding and topological characteristics of pre-miRs can potentially serve as discriminative measures in improving the designs and performances of current de novo predictors. We have incorporated the 13 features into the development of a new and better performing de novo classifier for identifying species-specific and nonconserved pre-miRs, wholly independent of phylogenetic conservation.

\section{MATERIALS AND METHODS}

\section{Biologically relevant data sets}

\section{Precursor miRNA sequences}

We retrieved 4028 curated pre-miRs spanning 45 species from miRBase Registry Database version 8.2 (Griffiths-Jones et al. 2006) as of July 2006. As strong sequence homologies exist among pre-miRs both within a single and between different species, the original data set was filtered to $90 \%$ identity using a greedy incremental clustering algorithm (Li and Godzik 2006). Briefly, all the sequences were first sorted in order of decreasing length, where the longest one became the representative of the first cluster. Each remaining sequence was compared with the existing representatives and grouped into their cluster if the similarity with any representative was above a given threshold (i.e., 0.9); otherwise, that sequence became the representative of a new cluster. Consequently, we analyzed 2241 nonredundant pre-miRs spanning 41 species categorized into arthropoda, nematoda, vertebrata, viridiplantae, and viruses (Table 1); none belonging to Gorilla gorilla, Macaca nemestrina, Pan paniscus, and Pongo pygmaeus were retained.

\section{Functional noncoding RNA sequences}

We retrieved all available seed ncRNA sequences from Rfam Database version 7.0 (Griffiths-Jones et al. 2005) as of March 2005. After removing 46 types of pre-miRs, we analyzed 12,387 curated seed ncRNAs spanning 457 types categorized into 16 families (Table 1). These prokaryotic and eukaryotic ncRNAs have a length distribution similar to the known pre-miRs, and can fold with hairpins or stem-loops (Eddy 2001; Storz 2002; Svoboda and Cara 2006). Briefly, cis-regulatory elements are a well-conserved untranslated mRNA leader region capable of adopting alternate structural conformations that result in transcription termination or transcription elongation into the downstream region. For example, the T-box leader regulates transcription of many bacterial aminoacyl-tRNA synthetases, amino acid biosynthesis, and amino acid transport genes using uncharged tRNA as the effector (Winkler et al. 2001). The internal ribosome entry site (IRES) is a nucleotide sequence that allows for translation initiation in the middle of an mRNA. It mimics the $5^{\prime}$-cap structure, critical for the assembly of the initiation complex. Riboswitches are highly conserved RNA regulatory elements, embedded within the $5^{\prime}$-untranslated region (UTR) of biosynthesis genes or operons, and cis modulate their expressions upon binding to metabolite (e.g., guanine and thiamine pyrophosphate), without involving protein cofactors (Hesselberth and Ellington 2002; Lai 2003; Stormo 2003; Winkler and Breaker 2003; Mandal and Breaker 2004; Nudler and Mironov 2004; Soukup and Soukup 2004; Vitreschak et al. 2004). Thermoregulators are cis-regulatory elements commonly found in the 5' UTR of mRNAs, whose secondary structure is regulated by temperature. For example, the structural motif of PrfA thermoregulator represses translation at $30^{\circ} \mathrm{C}$ by masking the ShineDalgarno sequence, but conformational change frees it for ribosome binding to allow maximal translation when the temperature rises to $37^{\circ} \mathrm{C}$ (Johansson et al. 2002). Anti-senses are characterized by a long hairpin structure interrupted by several unpaired residues or bulged loops, involved in negative regulation. For instance, the micF gene is a Escherichia coli stress response gene encoding an untranslated 93-nt anti-sense that binds to its target ompF mRNA (of the outer membrane porin gene) (Delihas and Forst 2001). It regulates ompF expression posttranscriptionally by causing translational repression. Ribozymes (e.g., the Hepatitis $\delta$-virus ribozyme and Hammerhead ribozyme) possess endonuclease function and catalyze a range of reactions such as self-cleavage of hepatitis $\delta$-virus transcript (PuertaFernandez et al. 2003). Small nucleolar RNAs (snoRNAs) can be functionally divided into C/D snoRNAs or H/ACA snoRNAs acting as guides for site-specific 2'-O-ribose methylation or as guides for pseudouridylation in the post-transcriptional processing of rRNAs (Weinstein and Steitz 1999). Spliceosomal RNAs (splicing RNAs), e.g., U1-2 and U4-6 (Storz et al. 2005), are small nuclear RNAs constituting the spliceosome that process premRNA into mRNA by excising the intronic regions. Transfer RNAs (tRNAs) exist as 54-93-nt hydrogen-bonded cloverleaf structures, involved in transporting amino acids to the site of protein synthesis during translation (Sprinzl and Vassilenko 2005). Group I/II intron RNAs are large self-splicing ribozymes catalyzing their own excision from mRNA, tRNA, and rRNA precursors (Cech 1990; Bonen and Vogel 2001).

\section{$m R N A$ sequences}

We investigated 31 mRNA sequences that tend to fold into complex RNA structures with extremely negative MFEs (Table 1; Freyhult et al. 2005). They were randomly selected from the GenBank DNA database (Benson et al. 2005), as previously reported (Freyhult et al. 2005). 


\section{Pseudohairpin sequences}

We analyzed 8494 pseudohairpins from the protein-coding regions (CDSs) according to the UCSC refGene annotation tables (Karolchik et al. 2003) and human RefSeq genes (Pruitt and Maglott 2001) without any known experimentally validated alternative splicing (AS) events, as described earlier (Xue et al. 2005). These genomic inverted repeats are analogous to but do not encode genuine human pre-miRs, by displaying similar distribution in terms of their length, hairpin structures, and MFEs. They possess $\geq 18 \mathrm{bp}$, including the GU wobble pairs, MFE $\leq-15 \mathrm{kcal} / \mathrm{mol}$, and fold without multiple loops in their RNA structures.

\section{Random sequences}

Four sets of $R=10^{4}$ shuffled or randomized RNAs, $\mathbf{r}_{n}=r_{1} r_{2} \ldots r_{L}$, serving as the genomic background, are synthesized from each $n$th native RNA sequence $\mathbf{s}_{n}=s_{1} s_{2} \ldots s_{L}$, using four sequence randomization algorithms. $L$ is the length of sequence in nucleotides and $s_{i} \in \sum=(A, C, G, U)$ is the biochemical nucleotide at the ith position.

Mononucleotide shuffling. We implemented the "Fisher-Yates shuffle algorithm" that sequentially swaps the mononucleotides at all positions of $\mathbf{s}_{n}$ with another at a randomly selected position. It consumes $\Theta(L \log L)$ bits and runs in linear time. The order of the shuffled nucleotides is truly random, preserving the mono- but not the dinucleotide frequencies.

1. Vars: $\mathbf{s}_{n} \leftarrow \mathbf{r}_{n}$.

2. For $i \leftarrow L: 1$, do

3. $j \leftarrow \operatorname{uniform}(1, i)$.

4. If $i \neq j$, then $\operatorname{swap}\left(r_{i}, r_{j}\right)$.

Dinucleotide shuffling. Previous algorithms (Workman and Krogh 1999; Bonnet et al. 2004b) were heuristically based, and the shuffled RNA sequences might not guarantee to preserve correctly the exact mono- and dinucleotide frequencies as the native RNA. We implemented the exact "Altschul-Erickson algorithm" (Altschul and Erickson 1985) such that it shuffles $\mathbf{s}_{n}$ while preserving exactly both the mono- and dinucleotide frequencies. The native and shuffled sequences always share the same first and last nucleotides (Coward 1999). The order of the shuffled nucleotides is "less random" due to fewer possible dinucleotide preserving permutations.

1. For each $r \in \mathbf{r}_{n}$, do

2. create an edge-list $L_{r}$ of edge-pairs $(r, x)$ with nucleotides $r$ and $x$ occurring as a dinucleotide $r x$ in $\mathbf{s}_{n}$.

3. For each $r \neq r_{L} \in \mathbf{r}_{n}$, do

4. $E\left(\mathbf{s}_{n}\right) \leftarrow$ select randomly an edge-pair from $L_{r}$. $E\left(\mathbf{s}_{n}\right)$ contains at most three edge-pairs.

5. $G \leftarrow(V, E)$ is the last-edge graph such that $(r, x) \in V$ and $(r, x) \in E\left(\mathbf{s}_{n}\right)$. If any vertex in $G$ is not connected to $r_{L}$, then go to (3). Else, go to (6) as all vertices are connected in $G$ to $r_{L}$.

6. For each $r \in \mathbf{r}_{n}$, do

7. permute the remaining edge-pairs in $L_{b}-E\left(\mathbf{s}_{n}\right), L_{r} \leftarrow L_{r}$ $\cup E\left(\mathbf{s}_{n}\right)$.

8. Vars: $r_{1} \leftarrow s_{1}$.
9. For $i \leftarrow 1: L-1$, do

10. generate $r_{i+1}$ such that $\left(r_{i}, r_{i+1}\right) \in L_{r}$.

Zero-order Markov model. A new random sequence $\mathbf{r}_{n}$ is formed by iteratively adding nucleotide $r_{i}$ sampled with expected mononucleotide frequencies $\mathrm{F}\left(\sum, \mathbf{s}_{n}\right)$. The sequence $\mathbf{r}_{n}$ is "truly" random, and its mononucleotide frequencies fluctuate about the native ones.

1. Compute $\mathrm{F}\left(\sum, \mathbf{s}_{n}\right)$ from $\mathbf{s}_{n}$.

2. For $i \leftarrow 1: L$, do

3. $r_{i} \leftarrow$ sampling with $\mathrm{F}\left(\sum, \mathbf{s}_{n}\right)$.

First-order Markov model. A new random sequence $\mathbf{r}_{n}$ is formed by first choosing a nucleotide $r_{1}$ sampled with expected mononucleotide frequencies $\mathrm{F}\left(\sum, \mathbf{s}_{n}\right)$. Iteratively add the next nucleotide $r_{i+1}$ sampled with conditional probabilities $\mathrm{P}\left(r_{i+1} \mid r_{i}\right)$; i.e., the probability of occurrence of a nucleotide at a particular position depends only on the previous nucleotide. The sequence is "truly" random, and its dinucleotide frequencies fluctuate around the native ones.

1. Compute $\mathrm{F}\left(\sum, \mathbf{s}_{n}\right)$ and $\mathrm{G}\left(\sum_{1}, \sum_{2}, \mathbf{s}_{n}\right)$ from $\mathbf{s}_{n}$.

2. $r_{1} \leftarrow$ sampling with $\mathrm{F}\left(\sum, \mathbf{s}_{n}\right)$.

3. For $i \leftarrow 2$ : $L$, do

4. $r_{i} \leftarrow$ sampling with $\mathrm{P}\left(\sum_{2} \mid \sum_{1}\right)=\mathrm{G}\left(\sum_{1}, \sum_{2}, \mathbf{s}_{n}\right) / \mathrm{F}\left(\sum_{1}, \mathbf{s}_{n}\right)$.

\section{RNA folding measures}

Normalized base-pairing propensity, $P(S)$, measures the total number of base pairs present in the RNA secondary structure $S$ normalized to the sequence length $L$ (Schultes et al. 1999). $P(S)$ removes the bias that a long sequence tends to have more base pairs. It ranges $[0.0,0.5], 0.0$ for no base-pair interactions and 0.5 for a maximum of $L / 2$ base pairs.

\section{Normalized minimum free energy of folding}

$\operatorname{MFE}(\mathbf{s})$, for sequence $\mathbf{s}$ is the lowest MFE for the most favorable conformation from a vast population of predicted RNA secondary structures, normalized to the sequence length $L$ (Seffens and Digby 1999; Freyhult et al. 2005). MFE(s) removes the bias that a long sequence tends to have lower MFE. Alternatively, adjusted MFE (AMFE) refers to MFE(s)×100 nt (Zhang et al. 2006b).

\section{MFE Index 1}

$M F E I_{1}$, is the ratio of $M F E(\mathbf{s})$ and $\% G+C$ content (Zhang et al. 2006b).

\section{Normalized Shannon entropy}

$Q(s)$ in Equation (1), characterizes the base-pairing probability distribution (BPPD) per base in a sequence $\mathbf{s}$ as a chaotic dynamical system (Huynen et al. 1997; Schultes et al. 1999; Freyhult et al. 2005). The local dominance of a single structure within the Boltzmann distribution of alternative secondary structures is strongly correlated with the reliability of the MFE structure. Low values of $Q(\mathbf{s})$ correspond to the BPPD that are dominated by 
a single or a few base-pairing probabilities. These bases are better predicted than those having multiple alternative states.

$$
\begin{aligned}
& Q(\mathbf{s})=-\frac{1}{L} \sum_{i<j} p_{i j} \log _{2}\left(p_{i j}\right) . \\
& p_{i j}=\sum_{S_{\alpha} \in S(\mathbf{s})} P\left(S_{\alpha}\right) \delta_{i j}^{\alpha}, P\left(S_{\alpha}\right)=\frac{e^{-E_{\alpha}} / R T}{\aleph}, \aleph=\sum_{S_{\alpha} \in S(\mathbf{s})} \frac{e^{-E_{\alpha}}}{R T} .
\end{aligned}
$$

Here, the McCaskill base-pair probability $p_{i j}$ is the probability of base-pairing between bases $i$ and $j . \delta_{i j}^{\alpha}=1$ if $x_{i}$ pairs with $x_{j}$, 0 otherwise. RNAs exist in vivo as an ensemble of secondary structures $S_{\alpha} \in \mathrm{S}(\mathbf{s})$ following the Boltzmann distribution probability $P\left(S_{\alpha}\right)$ (Mathews 2004).

\section{Normalized base-pair distance}

$D(\mathbf{s})$ in Equation (2), is the base-pair distance for all pairs of structures $S_{\alpha}$ and $S_{\beta}$ on s (Moulton et al. 2000; Freyhult et al. 2005).

$$
\begin{aligned}
D(\mathbf{s}) & =\frac{1}{2 L} \sum_{S_{\alpha}, S_{\beta} \in \mathbf{S}(\mathbf{x})}\left[P\left(S_{\alpha}\right) P\left(S_{\beta}\right) d_{B P}\left(S_{\alpha}, S_{\beta}\right)\right] \\
& =\frac{1}{L} \sum_{i<j} p_{i j}\left(1-p_{i j}\right) .
\end{aligned}
$$

Here, the number of base pairs not shared by them is given by $d_{B P}\left(S_{\alpha}, S_{\beta}\right)=\left|S_{\alpha} \cup S_{\beta}\right|\left|S_{\alpha} \cap S_{\beta}\right|=\sum_{i<j}\left(\delta_{i j}^{\alpha}+\delta_{i j}^{\beta}-2 \delta_{i j}^{\alpha} \delta_{i j}^{\beta}\right)$. The number

of base pairs in $S_{\alpha}$ is $\left|S_{\alpha}\right|=\sum_{i<j \delta_{i j}^{\alpha}}$. Definitions of $p_{i j}$ and $\delta_{i j}^{\alpha}$ follow those of $Q(\mathbf{s})$ in Equation (1).

\section{Second (or the Fiedler) eigenvalue}

$F(S)$ in Equation (3) measures the compactness of a tree-graph $G=(V, E)$ (Fera et al. 2004; Gan et al. 2004). At the coarsest scale, each vertex $v \in V$ represents a bulge loop, hairpin loop, internal loop, the $5^{\prime}$ and $3^{\prime}$ unpaired termini, or the multibranch loop; each edge $e \in E$ denotes an RNA stem. $F(S)$ is computed from the Laplacian matrix $\mathbf{L}(G)$, which is a mathematical representation of the tree-graph $G . F(S)$ can be used as a similarity measure among a collection of RNA secondary structures.

$$
\mathbf{L}(G) \mathbf{X}=\lambda \mathbf{X} \Leftrightarrow F(S)=\text { FidlerEigen }[\mathbf{L}(G)] .
$$

\section{MFE Index 2}

$M F E I_{2}$, is the ratio of $M F E(\mathbf{s})$ and the number of stems $S$, which are structural motifs containing more than three contiguous base pairs.

\section{$Z$ score of RNA folding measure}

The $Z$ score of the RNA folding measure is described in Equation (4). The $Z$ score $Z\left(\mathbf{s}_{\mathrm{n}}\right)$ for the structural biases observed in a native RNA is computed via a Monte Carlo randomization approach (Workman and Krogh 1999; Bonnet et al. 2004b; Clote et al. 2005). It normalizes the feature $S\left(\mathbf{s}_{n}\right)$ of $n$th native RNA sequence $\mathbf{s}_{n}$ in terms of the units of standard deviations by which $S\left(\mathbf{s}_{n}\right)$ differs from the mean of inferred $R=10^{4}$ randomized RNA sequences $\mathbf{r}_{n}$. The corresponding $Z$ scores of $\operatorname{MFE}(\mathbf{s}), Q(\mathbf{s}), D(\mathbf{s})$, $P(S)$, and $F(S)$ are denoted as $z G, z Q, z D, z P$, and $z F$.

$$
Z\left(\mathbf{s}_{n}\right)=\frac{S\left(\mathbf{s}_{n}\right)-\mu_{n}}{\sigma_{n}}, \sigma_{n}^{2}=\frac{1}{R-1} \sum_{i=1}^{R}\left[S_{i}\left(\mathbf{r}_{n}\right)-\mu_{n}\right]^{2} .
$$

Here $S_{i}\left(\mathbf{r}_{n}\right)$ is the computed feature for the $i$ th random RNA sequence of $\mathbf{r}_{n} ; \mu_{n}$ and $\sigma_{n}$ are the sample mean and the standard deviation of the feature $S\left(s_{n}\right)$ for $R$ random RNA sequences $\mathbf{r}_{n}$.

\section{Statistical analysis}

\section{Computing the RNA folding measures and Z scores}

The most favorable RNA secondary structure for a given sequence and its normalized minimum free energy of folding $\operatorname{MFE}(\mathbf{s})$ are determined via RNAfold included in Vienna RNA Package 1.4 (Hofacker 2003), an implementation of Zuker's free energy minimization algorithm (Zuker and Stiegler 1981; Zuker 2003) with Turner energy parameters (Mathews et al. 1999). From the predicted structure, the intrinsic folding quantitative measures $P(S), Q(\mathbf{s})$, and $D(\mathbf{s})$ are computed by the perl script genRNAStats.pl interfaced to the module RNAlib of Vienna RNA Package 1.4 (Hofacker 2003). The topological descriptors $S$ and $F(S)$ are determined using an algorithm RNAspectral (see Supplemental materials for details). The normalized variants $z P, z G, z Q, z D$, and $z F$ are computed in a similar manner using genRNARandomStats.pl, after generating the four sets of random RNA sequences with genRandomRNA.pl. All intensive computations are performed on three clusters of 192 dual-core computational nodes.

Statistical analysis measuring the differences inherent within pre-miRs' global structural and intrinsic stability features

To compare the data sets and compute the probability that the samples are drawn from the same distribution, we conduct either nonparametric Kruskal-Wallis one-way analysis of variance (ANOVA) or nonparametric Mann-Whitney-Wilcoxon (Wilcoxon rank-sum). The former tests for statistically significant differences in the median values $(p<0.001)$ among the experimental groups against the control are greater than would be expected by chance. To isolate the groups that differ from the control, Dunn's method of multiple comparisons test is conducted at $p<0.01$. It does not include an adjustment for ties but allows the sample sizes of the experimental groups to be different. The latter method tests for statistically significant differences in the median values between two experimental groups $(p<0.001)$. Unlike parametric statistical tests like Student's $t$-test, both ANOVA and Wilcoxon rank-sum compare the ranks of the data values instead of the actual data values. Thus, they are robust to samples drawn from populations with non-normal distribution or which have unequal variances (Systat SigmaPlot 9.0 and SigmaStat 3.11).

\section{Correlation of quantitative metrics}

To quantify the correlation between measures for native pre-miRs, the Pearson correlation coefficients $C_{p}(f, g)$ in Equation (5) are computed, statistically significant at $p<0.001$. We are aware that $C_{p}$ is not robust to outliers and to non-Gaussian distributions, as 
it assumes a pseudo-Gaussian distribution of the data set. Thus, we also validate the results of $C_{p}$ against those of nonparametric Spearman-rank $C_{s}$ (ranks based) and Kendall's $C_{k}$ (relative ranks based) correlation metrics. Both $C_{s}$ and $C_{k}$ are robust to samples containing outliers or drawn from populations with unequal variances, non-normality distribution, and nonlinearity (Mathworks Matlab 7.1).

$$
C_{p}(f, g)=\frac{(f-\bar{f}) \cdot(g-\bar{g})}{\|f-\bar{f}\|\|g-\bar{g}\|}
$$

\section{SUPPLEMENTAL DATA}

The Supplemental details on RNAspectral and Tables S1-3, as well as the data sets (Fasta format files), raw statistical results (tabdelimited format and Excel files), and source codes are publicly available at http://web.bii.a-star.edu.sg/ stanley/Publications. Use of source codes is free purely for nonprofit or academic purpose adhering to the GNU General Public License (GPL) at http:// www.gnu.org/copyleft/gpl.html.

\section{ACKNOWLEDGMENTS}

The authors are indebted to the Theoretical Biochemistry Group of the Institute for Theoretical Chemistry at the University of Vienna for their public release of the Vienna RNA software package, providing core components to our analysis. We thank the two anonymous reviewers for their thorough comments and constructive suggestions that greatly improved the quality and technical ideas developed in this article. Sincere appreciation to the technical team of BII's Clustering Group for their best effort in ensuring the three clusters of 192 dual-core computational nodes run smoothly. This work was supported by the Bioinformatics Institute. S.N.K.L. received Ph.D. scholarship funds from the Agency for Science, Technology and Research (A*STAR), Singapore. S.N.K.L. and S.K.M. conceived the initial ideas. S.N.K.L. designed and performed the experiments. S.N.K.L. and S.K.M. wrote this manuscript.

Received July 6, 2006; accepted November 7, 2006.

\section{REFERENCES}

Adai, A., Johnson, C., Mlotshwa, S., Archer-Evans, S., Manocha, V., Vance, V., and Sundaresan, V. 2005. Computational prediction of miRNAs in Arabidopsis thaliana. Genome Res. 15: 78-91.

Altschul, S.F. and Erickson, B.W. 1985. Significance of nucleotide sequence alignments: A method for random sequence permutation that preserves dinucleotide and codon usage. Mol. Biol. Evol. 2: $526-538$.

Ambros, V. 2001. microRNAs: Tiny regulators with great potential. Cell 107: 823-826.

Ambros, V., Bartel, B., Bartel, D.P., Burge, C.B., Carrington, J.C., Chen, X., Dreyfuss, G., Eddy, S.R., Griffiths-Jones, S., Marshall, M., et al. 2003. A uniform system for microRNA annotation. RNA 9: 277-279.
Anthony, A.M. and Peter, M.W. 2005. Plant and animal microRNAs: Similarities and differences. Funct. Integr. Genomics 5: 129-135.

Banerjee, D. and Slack, F. 2002. Control of developmental timing by small temporal RNAs: A paradigm for RNA-mediated regulation of gene expression. Bioessays 24: 119-129.

Barash, D. 2003. Deleterious mutation prediction in the secondary structure of RNAs. Nucleic Acids Res. 31: 6578-6584.

Barash, D. 2004. Spectral decomposition for the search and analysis of RNA secondary structure. J. Comput. Biol. 11: 1169-1174.

Bartel, D.P. 2004. MicroRNAs: Genomics, biogenesis, mechanism, and function. Cell 116: 281-297.

Benson, D.A., Karsch-Mizrachi, I., Lipman, D.J., Ostell, J., and Wheeler, D.L. 2005. GenBank. Nucleic Acids Res. 33: D34-D38.

Bentwich, I., Avniel, A., Karov, Y., Aharonov, R., Gilad, S., Barad, O., Barzilai, A., Einat, P., Einav, U., Meiri, E., et al. 2005. Identification of hundreds of conserved and nonconserved human microRNAs. Nat. Genet. 37: 766-770.

Berezikov, E., Guryev, V., van de, B.J., Wienholds, E., Plasterk, R.H., and Cuppen, E. 2005. Phylogenetic shadowing and computational identification of human microRNA genes. Cell 120: 21-24.

Berezikov, E., Cuppen, E., and Plasterk, R.H. 2006. Approaches to microRNA discovery. Nat. Genet. (Suppl) 38: S2-S7.

Bonen, L. and Vogel, J. 2001. The ins and outs of group II introns. Trends Genet. 17: 322-331.

Bonnet, E., Wuyts, J., Rouze, P., and Van de Peer, Y. 2004a. Detection of 91 potential conserved plant microRNAs in Arabidopsis thaliana and Oryza sativa identifies important target genes. Proc. Natl. Acad. Sci. 101: 11511-11516.

Bonnet, E., Wuyts, J., Rouze, P., and Van de Peer, Y. 2004b. Evidence that microRNA precursors, unlike other non-coding RNAs, have lower folding free energies than random sequences. Bioinformatics 20: 2911-2917.

Brennecke, J., Hipfner, D.R., Stark, A., Russell, R.B., and Cohen, S.M. 2003. bantam encodes a developmentally regulated microRNA that controls cell proliferation and regulates the proapoptotic gene hid in Drosophila. Cell 113: 25-36.

Brenner, S., Johnson, M., Bridgham, J., Golda, G., Lloyd, D.H., Johnson, D., Luo, S., McCurdy, S., Foy, M., Ewan, M., et al. 2000. Gene expression analysis by massively parallel signature sequencing (MPSS) on microbead arrays. Nat. Biotechnol. 18: 630634.

Cech, T.R. 1990. Self-splicing of group I introns. Annu. Rev. Biochem. 59: $543-568$.

Chen, C.Z., Li, L., Lodish, H.F., and Bartel, D.P. 2004. MicroRNAs modulate hematopoietic lineage differentiation. Science 303: 83-86.

Clote, P. 2005. RNALOSS: A web server for RNA locally optimal secondary structures. Nucleic Acids Res. 33: W600-W604.

Clote, P., Ferre, F., Kranakis, E., and Krizanc, D. 2005. Structural RNA has lower folding energy than random RNA of the same dinucleotide frequency. RNA 11: 578-591.

Coward, E. 1999. Shufflet: Shuffling sequences while conserving the k-let counts. Bioinformatics 15: 1058-1059.

Cullen, B.R. 2004. Transcription and processing of human microRNA precursors. Mol. Cell 16: 861-865.

Cummins, J.M., He, Y., Leary, R.J., Pagliarini, R., Diaz Jr., L.A., Sjoblom, T., Barad, O., Bentwich, Z., Szafranska, A.E., Labourier, E., et al. 2006. The colorectal microRNAome. Proc. Natl. Acad. Sci. 103: 3687-3692.

Delihas, N. and Forst, S. 2001. MicF: An anti-sense RNA gene involved in response of Escherichia coli to global stress factors. J. Mol. Biol. 313: 1-12.

Doench, J.G. and Sharp, P.A. 2004. Specificity of microRNA target selection in translational repression. Genes \& Dev. 18: 504-511.

Eddy, S.R. 2001. Non-coding RNA genes and the modern RNA world. Nat. Rev. Genet. 2: 919-929.

Fera, D., Kim, N., Shiffeldrim, N., Zorn, J., Laserson, U., Gan, H., and Schlick, T. 2004. RAG: RNA-As-Graphs web resource. BMC Bioinformatics 5: 88. 
Freyhult, E., Gardner, P., and Moulton, V. 2005. A comparison of RNA folding measures. BMC Bioinformatics 6: 241.

Fu, H., Tie, Y., Xu, C., Zhang, Z., Zhu, J., Shi, Y., Jiang, H., Sun, Z., and Zheng, X. 2005. Identification of human fetal liver miRNAs by a novel method. FEBS Lett. 579: 3849-3854.

Gan, H.H., Fera, D., Zorn, J., Shiffeldrim, N., Tang, M., Laserson, U., Kim, N., and Schlick, T. 2004. RAG: RNA-As-Graphs databaseConcepts, analysis, and features. Bioinformatics 20: 1285-1291.

Grad, Y., Aach, J., Hayes, G.D., Reinhart, B.J., Church, G.M., Ruvkun, G., and Kim, J. 2003. Computational and experimental identification of C. elegans microRNAs. Mol. Cell 11: 1253 1263.

Grey, F., Antoniewicz, A., Allen, E., Saugstad, J., McShea, A., Carrington, J.C., and Nelson, J. 2005. Identification and characterization of human cytomegalovirus-encoded microRNAs. J. Virol. 79: 12095-12099.

Griffiths-Jones, S., Moxon, S., Marshall, M., Khanna, A., Eddy, S.R., and Bateman, A. 2005. Rfam: Annotating non-coding RNAs in complete genomes. Nucleic Acids Res. 33: D121-D124.

Griffiths-Jones, S., Grocock, R.J., van Dongen, S., Bateman, A., and Enright, A.J. 2006. miRBase: MicroRNA sequences, targets, and gene nomenclature. Nucleic Acids Res. 34: D140-D144.

Hertel, J. and Stadler, P.F. 2006. Hairpins in a haystack: Recognizing microRNA precursors in comparative genomics data. Bioinformatics 22: e197-e202.

Hesselberth, J.R. and Ellington, A.D. 2002. A (ribo) switch in the paradigms of genetic regulation. Nat. Struct. Biol. 9: 891-893.

Hofacker, I.L. 2003. Vienna RNA secondary structure server. Nucleic Acids Res. 31: 3429-3431.

Huynen, M., Gutell, R., and Konings, D. 1997. Assessing the reliability of RNA folding using statistical mechanics. J. Mol. Biol. 267: 11041112.

Johansson, J., Mandin, P., Renzoni, A., Chiaruttini, C., Springer, M., and Cossart, P. 2002. An RNA thermosensor controls expression of virulence genes in Listeria monocytogenes. Cell 110: 551-561.

Johnston, R.J. and Hobert, O. 2003. A microRNA controlling left/ right neuronal asymmetry in Caenorhabditis elegans. Nature 426: 845-849.

Jones-Rhoades, M.W. and Bartel, D.P. 2004. Computational identification of plant microRNAs and their targets, including a stressinduced miRNA. Mol. Cell 14: 787-799.

Karolchik, D., Baertsch, R., Diekhans, M., Furey, T.S., Hinrichs, A., Lu, Y.T., Roskin, K.M., Schwartz, M., Sugnet, C.W., Thomas, D.J., et al. 2003. The UCSC Genome Browser Database. Nucleic Acids Res. 31: 51-54.

Kim, V.N. 2005. MicroRNA biogenesis: Coordinated cropping and dicing. Nat. Rev. Mol. Cell Biol. 6: 376-385.

Lagos-Quintana, M., Rauhut, R., Lendeckel, W., and Tuschl, T. 2001. Identification of novel genes coding for small expressed RNAs. Science 294: 853-858.

Lagos-Quintana, M., Rauhut, R., Yalcin, A., Meyer, J., Lendeckel, W., and Tuschl, T. 2002. Identification of tissue-specific microRNAs from mouse. Curr. Biol. 12: 735-739.

Lai, E.C. 2003. RNA sensors and riboswitches: Self-regulating messages. Curr. Biol. 13: R285-R291.

Lai, E., Tomancak, P., Williams, R., and Rubin, G. 2003. Computational identification of Drosophila microRNA genes. Genome Biol. 4: R42.

Lau, N.C., Lim, L.P., Weinstein, E.G., and Bartel, D.P. 2001. An abundant class of tiny RNAs with probable regulatory roles in Caenorhabditis elegans. Science 294: 858-862.

Lee, R.C. and Ambros, V. 2001. An extensive class of small RNAs in Caenorhabditis elegans. Science 294: 862-864.

Lee, Y., Jeon, K., Lee, J.T., Kim, S., and Kim, V.N. 2002. MicroRNA maturation: Stepwise processing and subcellular localization. EMBO J. 21: 4663-4670.

Li, W. and Godzik, A. 2006. Cd-hit: A fast program for clustering and comparing large sets of protein or nucleotide sequences. Bioinformatics 22: 1658-1659.
Li, X. and Zhang, Y.Z. 2005. Computational detection of microRNAs targeting transcription factor genes in Arabidopsis thaliana. Comput. Biol. Chem. 29: 360-367.

Lim, L.P., Glasner, M.E., Yekta, S., Burge, C.B., and Bartel, D.P. 2003a. Vertebrate microRNA genes. Science 299: 1540.

Lim, L.P., Lau, N.C., Weinstein, E.G., Abdelhakim, A., Yekta, S., Rhoades, M.W., Burge, C.B., and Bartel, D.P. 2003b. The microRNAs of Caenorhabditis elegans. Genes \& Dev. 17: 991-1008.

Mallory, A.C. and Vaucheret, H. 2004. MicroRNAs: Something important between the genes. Curr. Opin. Plant Biol. 7: 120-125.

Mandal, M. and Breaker, R.R. 2004. Gene regulation by riboswitches. Nat. Rev. Mol. Cell Biol. 5: 451-463.

Mathews, D.H. 2004. Using an RNA secondary structure partition function to determine confidence in base pairs predicted by free energy minimization. RNA 10: 1178-1190.

Mathews, D.H., Sabina, J., Zuker, M., and Turner, D.H. 1999. Expanded sequence dependence of thermodynamic parameters improves prediction of RNA secondary structure. J. Mol. Biol. 288: 911-940.

Moss, E.G., Lee, R.C., and Ambros, V. 1997. The cold shock domain protein LIN-28 controls developmental timing in C. elegans and is regulated by the lin-4 RNA. Cell 88: 637-646.

Moulton, V., Zuker, M., Steel, M., Pointon, R., and Penny, D. 2000. Metrics on RNA secondary structures. J. Comput. Biol. 7: 277-292.

Nam, J.W., Shin, K.R., Han, J., Lee, Y., Kim, V.N., and Zhang, B.T. 2005. Human microRNA prediction through a probabilistic co-learning model of sequence and structure. Nucleic Acids Res. 33: 3570-3581.

Nudler, E. and Mironov, A.S. 2004. The riboswitch control of bacterial metabolism. Trends Biochem. Sci. 29: 11-17.

Pervouchine, D.D., Graber, J.H., and Kasif, S. 2003. On the normalization of RNA equilibrium free energy to the length of the sequence. Nucleic Acids Res. 31: e49.

Pfeffer, S., Zavolan, M., Grasser, F.A., Chien, M., Russo, J.J., Ju, J., John, B., Enright, A.J., Marks, D., Sander, C., et al. 2004. Identification of virus-encoded microRNAs. Science 304: 734-736.

Pfeffer, S., Sewer, A., Lagos-Quintana, M., Sheridan, R., Sander, C., Grasser, F.A., van Dyk, L.F., Ho, C.K., Shuman, S., Chien, M., et al. 2005. Identification of microRNAs of the herpesvirus family. Nat. Methods 2: 269-276.

Pruitt, K.D. and Maglott, D.R. 2001. RefSeq and LocusLink: NCBI gene-centered resources. Nucleic Acids Res. 29: 137-140.

Puerta-Fernandez, E., Romero-Lopez, C., Barroso-delJesus, A., and Berzal-Herranz, A. 2003. Ribozymes: Recent advances in the development of RNA tools. FEMS Microbiol. Rev. 27: 75-97.

Reinhart, B.J., Slack, F.J., Basson, M., Pasquinelli, A.E., Bettinger, J.C., Rougvie, A.E., Horvitz, H.R., and Ruvkun, G. 2000. The 21nucleotide let-7 RNA regulates developmental timing in Caenorhabditis elegans. Nature 403: 901-906.

Rivas, E. and Eddy, S.R. 2000. Secondary structure alone is generally not statistically significant for the detection of noncoding RNAs. Bioinformatics 16: 583-605.

Rodriguez, A., Griffiths-Jones, S., Ashurst, J.L., and Bradley, A. 2004. Identification of mammalian microRNA host genes and transcription units. Genome Res. 14: 1902-1910.

Samols, M.A., Hu, J., Skalsky, R.L., and Renne, R. 2005. Cloning and identification of a microRNA cluster within the latency-associated region of Kaposi's sarcoma-associated herpesvirus. J. Virol. 79: 9301-9305.

Sarnow, P., Jopling, C.L., Norman, K.L., Schutz, S., and Wehner, K.A. 2006. MicroRNAs: Expression, avoidance and subversion by vertebrate viruses. Nat. Rev. Microbiol. 4: 651-659.

Schultes, E.A., Hraber, P.T., and LaBean, T.H. 1999. Estimating the contributions of selection and self-organization in RNA secondary structure. J. Mol. Evol. 49: 76-83.

Seffens, W. and Digby, D. 1999. mRNAs have greater negative folding free energies than shuffled or codon choice randomized sequences. Nucleic Acids Res. 27: 1578-1584.

Sewer, A., Paul, N., Landgraf, P., Aravin, A., Pfeffer, S., Brownstein, M., Tuschl, T., van Nimwegen, E., and Zavolan, M. 
2005. Identification of clustered microRNAs using an ab initio prediction method. BMC Bioinformatics 6: 267.

Soukup, J.K. and Soukup, G.A. 2004. Riboswitches exert genetic control through metabolite-induced conformational change. Curr. Opin. Struct. Biol. 14: 344-349.

Sprinzl, M. and Vassilenko, K.S. 2005. Compilation of tRNA sequences and sequences of tRNA genes. Nucleic Acids Res. 33: D139-D140.

Stormo, G.D. 2003. New tricks for an old dogma: Riboswitches as cis-only regulatory systems. Mol. Cell 11: 1419-1420.

Storz, G. 2002. An expanding universe of noncoding RNAs. Science 296: $1260-1263$.

Storz, G., Altuvia, S., and Wassarman, K.M. 2005. An abundance of RNA regulators. Annu. Rev. Biochem. 74: 199-217.

Svoboda, P. and Cara, A.D. 2006. Hairpin RNA: A secondary structure of primary importance. Cell. Mol. Life Sci. 63: 901-908.

Tang, G. 2005. siRNA and miRNA: An insight into RISCs. Trends Biochem. Sci. 30: 106-114.

Tijsterman, M. and Plasterk, R.H. 2004. Dicers at RISC; the mechanism of RNAi. Cell 117: 1-3.

Vitreschak, A.G., Rodionov, D.A., Mironov, A.A., and Gelfand, M.S. 2004. Riboswitches: The oldest mechanism for the regulation of gene expression? Trends Genet. 20: 44-50.

Wang, X.J., Reyes, J., Chua, N.H., and Gaasterland, T. 2004. Prediction and identification of Arabidopsis thaliana microRNAs and their mRNA targets. Genome Biol. 5: R65.

Wang, X., Zhang, J., Li, F., Gu, J., He, T., Zhang, X., and Li, Y. 2005. MicroRNA identification based on sequence and structure alignment. Bioinformatics 21: 3610-3614.

Washietl, S. and Hofacker, I.L. 2004. Consensus folding of aligned sequences as a new measure for the detection of functional RNAs by comparative genomics. J. Mol. Biol. 342: 19-30.

Weinstein, L.B. and Steitz, J.A. 1999. Guided tours: From precursor snoRNA to functional snoRNP. Curr. Opin. Cell Biol. 11: 378-384.

Wheeler, G., Ntounia-Fousara, S., Granda, B., Rathjen, T., and Dalmay, T. 2006. Identification of new central nervous system specific mouse microRNAs. FEBS Lett. 580: 2195-2200.
Winkler, W.C. and Breaker, R.R. 2003. Genetic control by metabolitebinding riboswitches. ChemBioChem 4: 1024-1032.

Winkler, W.C., Grundy, F.J., Murphy, B.A., and Henkin, T.M. 2001. The GA motif: An RNA element common to bacterial antitermination systems, rRNA, and eukaryotic RNAs. RNA 7: 1165-1172.

Workman, C. and Krogh, A. 1999. No evidence that mRNAs have lower folding free energies than random sequences with the same dinucleotide distribution. Nucleic Acids Res. 27: 48164822.

Xu, P., Vernooy, S.Y., Guo, M., and Hay, B.A. 2003. The Drosophila MicroRNA Mir-14 suppresses cell death and is required for normal fat metabolism. Curr. Biol. 13: 790-795.

Xue, C., Li, F., He, T., Liu, G.P., Li, Y., and Zhang, X. 2005. Classification of real and pseudo microRNA precursors using local structure-sequence features and support vector machine. BMC Bioinformatics 6: 310 .

Yekta, S., Shih, I.H., and Bartel, D.P. 2004. MicroRNA-directed cleavage of HOXB8 mRNA. Science 304: 594-596.

Yousef, M., Nebozhyn, M., Shatkay, H., Kanterakis, S., Showe, L.C., and Showe, M.K. 2006. Combining multi-species genomic data for microRNA identification using a Naive Bayes classifier. Bioinformatics 22: 1325-1334.

Zeng, Y. and Cullen, B.R. 2004. Structural requirements for premicroRNA binding and nuclear export by Exportin 5. Nucleic Acids Res. 32: 4776-4785.

Zhang, B., Pan, X., Cobb, G.P., and Anderson, T.A. 2006a. Plant microRNA: A small regulatory molecule with big impact. Dev. Biol. 289: 3-16.

Zhang, B., Pan, X., Cox, S., Cobb, G., and Anderson, T. $2006 \mathrm{~b}$. Evidence that miRNAs are different from other RNAs. Cell. Mol. Life Sci. 63: 246-254.

Zuker, M. 2003. Mfold web server for nucleic acid folding and hybridization prediction. Nucleic Acids Res. 31: 3406-3415.

Zuker, M. and Stiegler, P. 1981. Optimal computer folding of large RNA sequences using thermodynamics and auxiliary information. Nucleic Acids Res. 9: 133-148. 

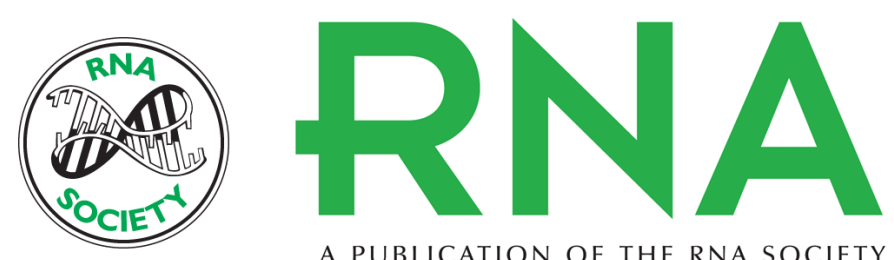

A PUBLICATION OF THE RNA SOCIETY

\section{Unique folding of precursor microRNAs: Quantitative evidence and implications for de novo identification}

Stanley NG Kwang Loong and Santosh K. Mishra

RNA 2007 13: 170-187 originally published online December 28, 2006

Access the most recent version at doi:10.1261/rna.223807

\section{References This article cites 103 articles, 21 of which can be accessed free at: http://rnajournal.cshlp.org/content/13/2/170.full.html\#ref-list-1}

\section{License}
Email Alerting Receive free email alerts when new articles cite this article - sign up in the box at the Service top right corner of the article or click here.

To subscribe to $R N A$ go to:

http://rnajournal.cshlp.org/subscriptions 